MATHEMATICS OF COMPUTATION

Volume 72, Number 241, Pages 307-333

S 0025-5718(02)01423-0

Article electronically published on May 3, 2002

\title{
CONVERGENCE STUDY OF THE CHORIN-MARSDEN FORMULA
}

\author{
LUNG-AN YING
}

\begin{abstract}
Using the fundamental solution of the heat equation, we give an expression of the solutions to two-dimensional initial-boundary value problems of the Navier-Stokes equations, where the vorticity is expressed in terms of a Poisson integral, a Newtonian potential, and a single layer potential. The density of the single layer potential is the solution to an integral equation of Volterra type along the boundary. We prove there is a unique solution to the integral equation. One fractional time step approximation is given, based on this expression. Error estimates are obtained for linear and nonlinear problems. The order of convergence is $\frac{1}{4}$ for the Navier-Stokes equations. The result is in the direction of justifying the Chorin-Marsden formula for vortex methods. It is shown that the density of the vortex sheet is twice the tangential velocity for the half plane, while in general the density differs from it by one additional term.
\end{abstract}

\section{INTRODUCTION}

The convergence and stability problems for the vortex method have been studied by many authors. Usually a fractional steps approach is applied in the vortex method for viscous flows; therefore viscous splitting is a basic problem in the mathematical theory. The convergence problem of viscous splitting was first studied in [2], and since then there have been quite a number of works on this subject (see [10] and references therein).

Vorticity is the main variable in the computation of the vortex method. However, a velocity boundary condition is applied on a solid boundary and the boundary condition for vorticity is indirect. To approximate the velocity boundary condition, it was proposed that the velocity field be extended oddly after each convection step; then one vortex sheet was created, which resulted in an approximate zero velocity on the boundary (see [4]). This scheme was formulated as

$$
\omega_{i}=\left(H_{k} \circ \phi \circ E_{k}\right)^{i} \omega_{0},
$$

where $k>0$ is the length of temporal steps, $\omega_{i}$ is the vorticity at $t_{i}=i k, \omega_{0}$ is the initial data, $E_{k}$ is the solver of the Euler equation, $H_{k}$ is the solver of the heat equation on the entire space, and $\phi$ is the "vorticity creation operator". Stability and convergence of (1) for linear equations was proved in [5].

Convergence of the Chorin-Marsden formula for a half plane domain was proved in [3], with a slight modification, where the tangential component of velocity was

Received by the editor June 5, 2000 and, in revised form, January 3, 2001.

2000 Mathematics Subject Classification. Primary 65M99; Secondary 35Q30, 76D05, 76M25.

Key words and phrases. Vortex method, Navier-Stokes equation, fractional step method. 
extended oddly and the normal component was extended evenly. An error estimate with upper bound $O\left(k^{\frac{1}{4}}\right)$ was obtained. Partial analysis for convex domains was gicen in [9, also with some modification, where the extended velocity field was multiplied by a Jacobian determinant. It was observed (see 9]) that both odd extension and even extension of the normal component of velocity yielded convergent results for the half plane domain. We notice that the way to create a vortex sheet is not unique, but all of them should be close enough so that the differences between them approach zero as $k \rightarrow 0$.

The aim of this paper is to study the problem for general bounded domains. The idea is to derive an expression of the density of vortex sheets for exact solutions, then make a time discretization to obtain an approximate one. In our scheme the derivation of vortex sheet is indirect, since it satisfies an integral equation of Volterra type along the boundary. A direct one may be possible. However, since we have the exact expression, the approximate one is required to be consistent with it. In [5], [3], [9] the density of the vortex sheet equals twice the tangential velocity after each convection step. We will make a remark in the final section, indicating that the density of the vortex sheet of our scheme is the same if the domain is a half plane. However, in the general case they are different by one additional term, which does not vanish in general. We hope this additional term approaches zero as $k \rightarrow 0$ for some cases. This will be the subject of our further study.

In order to state our scheme and convergence result, let us write down the equations first. Let $\Omega$ be a bounded domain in $\mathrm{R}^{2}$, and $\partial \Omega$ its boundary. The initialboundary value problems of the Navier-Stokes equations for viscous incompressible flow are

$$
\begin{gathered}
\frac{\partial u}{\partial t}+(u \cdot \nabla) u+\nabla p=\triangle u, \\
\nabla \cdot u=0, \\
\left.u\right|_{x \in \partial \Omega}=0,\left.\quad u\right|_{t=0}=u_{0},
\end{gathered}
$$

where $u$ is the velocity and $p$ is the pressure. Without loss of generality we set the density and viscosity equal to one. Let $\nabla \wedge=\left(\frac{\partial}{\partial x_{2}},-\frac{\partial}{\partial x_{1}}\right)$, and let the vorticity $\omega=-\nabla \wedge u$; then the vorticity formulation of the Navier-Stokes equation is

$$
\begin{gathered}
\frac{\partial \omega}{\partial t}+u \cdot \nabla \omega=\triangle \omega, \\
-\triangle \psi=\omega, \quad u=\nabla \wedge \psi, \\
\left.\psi\right|_{x \in \partial \Omega}=0, \\
\left.u\right|_{x \in \partial \Omega}=0, \\
\left.\omega\right|_{t=0}=\omega_{0}=-\nabla \wedge u_{0} .
\end{gathered}
$$

Let $\rho(\xi, t), \xi \in \partial \Omega, t \in[0, T]$ be the density of the vortex sheet of an exact solution; then the contribution of $\rho$ to the solution is

$$
\int_{0}^{t} \int_{\partial \Omega} K(x-\xi, t-\tau) \rho(\xi, \tau) d s_{\xi} d \tau
$$


where $K$ is the fundamental solution of the heat equation:

$$
K(x, t)=\frac{1}{4 \pi t} e^{-\frac{|x|^{2}}{4 t}} .
$$

Let $F=u \cdot \nabla \omega$, and we extend $\omega_{0}$ smoothly to $\mathrm{R}^{2}$ so that it is compactly supported; then we will prove that the solution to (5)-(9) can be expressed in terms of the sum of a Poisson integral, a Newtonian potential, and (10):

$$
\begin{aligned}
\omega & =\int_{\mathrm{R}^{2}} K(x-\xi, t) \omega_{0}(\xi) d \xi-\int_{0}^{t} \int_{\Omega} K(x-\xi, t-\tau) F(\xi, \tau) d \xi d \tau \\
& +\int_{0}^{t} \int_{\partial \Omega} K(x-\xi, t-\tau) \rho(\xi, \tau) d s_{\xi} d \tau
\end{aligned}
$$

For exact solutions the velocity vanishes on the boundary, so $\rho$ cannot be expressed in terms of velocity. Instead we will prove that $\rho$ satisfies

$$
\int_{0}^{t} \int_{\partial \Omega} L(\eta, \xi, t-\tau) \rho(\xi, \tau) d s_{\xi} d \tau+f(\eta, t)=0
$$

where

$$
\begin{aligned}
f(\eta, t) & =\int_{\Omega} M(x, \eta)\left\{\int_{\mathrm{R}^{2}} K(x-\xi, t) \omega_{0}(\xi) d \xi\right. \\
& \left.-\int_{0}^{t} \int_{\Omega} K(x-\xi, t-\tau) F(\xi, \tau) d \xi d \tau\right\} d x,
\end{aligned}
$$

and the kernels $L, M$ will be given later.

The following scheme is essentially the same as (1), except the method of vorticity creation is different. By induction we assume that the approximate solution $\omega_{k}(t)$ is already known for $t<t_{i}, t_{i}=i k, i=0,1, \cdots$; then $\omega_{k}(t)$ is obtained in $\left[t_{i}, t_{i+1}\right)$ by the following fractional time step scheme.

Convection step. Solve the inviscid problem

$$
\begin{gathered}
\frac{\partial \tilde{\omega}_{k}}{\partial t}+\tilde{u}_{k} \cdot \nabla \tilde{\omega}_{k}=0, \quad x \in \Omega, \quad t \in\left[t_{i}, t_{i+1}\right), \\
-\triangle \tilde{\psi}_{k}=\tilde{\omega}_{k}, \quad \tilde{u}_{k}=\nabla \wedge \tilde{\psi}_{k}, \quad x \in \Omega, \quad t \in\left[t_{i}, t_{i+1}\right), \\
\left.\tilde{\psi}_{k}\right|_{x \in \partial \Omega}=0, \\
\tilde{\omega}_{k}\left(t_{i}\right)=\omega_{k}\left(t_{i}-0\right),
\end{gathered}
$$

where $\omega_{k}(-0)=\omega_{0}$.

Vorticity creation step. Solve

$$
\int_{0}^{t} \int_{\partial \Omega} L(\eta, \xi, t-\tau) \rho_{k}(\xi, \tau) d s_{\xi} d \tau+f_{k}(\eta, t)=0
$$

where $\rho_{k}(\xi, t)$ is obtained for $t \in\left[0, t_{i}\right)$ by the previous steps and $f_{k}$ is a linear interpolation

$$
f_{k}(\eta, \tau)=\frac{1}{k}\left(\left(t_{i+1}-\tau\right) f_{k i}+\left(\tau-t_{i}\right) f_{k i+1}\right), \quad \tau \in\left[t_{i}, t_{i+1}\right],
$$


and $f_{k i}, f_{k i+1}$ is given by

$$
\begin{aligned}
f_{k i}(\eta)=\int_{\Omega} M(x, \eta)\{ & \int_{\mathrm{R}^{2}} K\left(x-\xi, t_{i}\right) \omega_{0}(\xi) d \xi \\
& \left.-\sum_{j=0}^{i-1} \int_{\Omega} K\left(x-\xi, t_{i}-t_{j}\right) \int_{t_{j}}^{t_{j+1}} F(\xi, \tau) d \xi d \tau\right\} d x,
\end{aligned}
$$

where $F=\tilde{u}_{k} \cdot \nabla \tilde{\omega}_{k}$.

Diffusion step. Solve the heat equation

$$
\frac{\partial \omega_{k}}{\partial t}=\triangle \omega_{k}, \quad x \in \mathrm{R}^{2}, \quad t \in\left[t_{i}, t_{i+1}\right),
$$

with initial data

$$
\omega_{k}\left(t_{i}\right)=\omega_{k}\left(t_{i}-0\right)+\omega_{1}^{\prime}+\omega_{2}^{\prime},
$$

where

$$
\omega_{1}^{\prime}(x)=\left\{\begin{array}{lr}
\tilde{\omega}_{k}\left(t_{i+1}-0\right)-\omega_{k}\left(t_{i}-0\right), & x \in \Omega, \\
0, & x \notin \Omega,
\end{array}\right.
$$

and

$$
\omega_{2}^{\prime}\left(\xi+s_{n} n_{\xi}\right)=\int_{t_{i}}^{t_{i+1}} \rho_{k}(\xi, t) d t \delta\left(s_{n}\right), \quad \forall \xi \in \partial \Omega,-\infty<s_{n}<+\infty,
$$

where $n_{\xi}$ is the outward unit normal vector at $\xi$, and $\delta$ is the Dirac $\delta$-function. More precisely,

$$
\left\langle\omega_{2}^{\prime}, \varphi\right\rangle=\int_{\partial \Omega}\left(\int_{t_{i}}^{t_{i+1}} \rho_{k}(\xi, t) d t\right) \varphi(\xi) d s_{\xi}, \quad \forall \varphi \in C_{0}^{\infty}\left(\mathrm{R}^{2}\right) .
$$

We are now ready to state our convergence and error estimate results. For simplicity we assume that the domain $\Omega$ is simply connected, $\partial \Omega$ is sufficiently smooth, and the solution to (2)-(4) is sufficiently smooth. We will use the usual notations of Sobolev spaces, $H^{s}(\Omega)$, and norms, $\|\cdot\|_{s}$, where $s$ is a real number.

Theorem 1.1. If $1<\sigma<\frac{3}{2}$, then

$$
\left\|\left(\omega-\omega_{k}\right)\left(t_{i}-0\right)\right\|_{\sigma-1} \leq C^{*} k^{\frac{1}{4}}
$$

for $0 \leq i \leq\left[\frac{T}{k}\right]$, provided $k \leq k_{0}$, where $k_{0}>0$ is a fixed constant and $C^{*}$ is a constant independent of $k$.

We remark that the rate of $\frac{1}{4}$ is the same as that in [3], but the norm is different.

We will derive the expression (11) and the equation (12) for vortex sheets in the next section. Using those expressions, we will study the approximate scheme (14)(22) in Section 3. We will estimate the upper bound and error for linear problems in Section 4 and Section 5 , then study nonlinear problems in Section 6 . Finally we will make a remark on the vortex sheet in Section 7 . Throughtout this paper we will always denote by $C$ a generic positive constant, and $C_{0}, C_{1}, \cdots, M_{0}, M_{1}, \cdots$, will be different positive constants. 


\section{An EXPRESSION OF THE EXACT SOLUTIONS}

To derive an equivalent formulation of (5)-(9) we prove

Lemma 2.1. Let $\omega \in L^{2}(\Omega)$ and $\psi \in H_{0}^{1}(\Omega) \cap H^{2}(\Omega)$ satisfy

$$
-\triangle \psi=\omega, \quad x \in \Omega .
$$

Then $\left.\nabla \psi\right|_{\partial \Omega}=0$ if and only if $\int_{\Omega} \omega h d x=0$ for all harmonic functions $h \in H^{1}(\Omega)$.

Proof. We denote by $n$ the outward unit normal vector on $\partial \Omega$, and by $\nu$ the anticlockwise unit tangential vector on $\partial \Omega$. We have

$$
\begin{aligned}
\int_{\Omega} \omega h d x=-\int_{\Omega} \triangle \psi h d x & =\int_{\Omega} \nabla \psi \cdot \nabla h d x-\int_{\partial \Omega} \frac{\partial \psi}{\partial n} h d s \\
& =-\int_{\partial \Omega} \frac{\partial \psi}{\partial n} h d s .
\end{aligned}
$$

Since $\left.h\right|_{\partial \Omega} \in H^{1 / 2}(\partial \Omega)$ is arbitrary, $\left.\frac{\partial \psi}{\partial n}\right|_{\partial \Omega}=0$ is equivalent to $\int_{\Omega} \omega h d x=0$, and $\left.\frac{\partial \psi}{\partial \nu}\right|_{\partial \Omega}=0$ is obvious.

We set $H(\Omega)=\left\{h \in H^{1}(\Omega) ; \triangle h=0\right\}$; then (5)-(9) is equivalent to (5)-(7), (9) and

$$
\int_{\Omega} \omega h d x=0, \quad \forall h \in H(\Omega)
$$

Let us express a harmonic function in terms of a double layer potential. We define

$$
M(x, \eta)=\frac{x-\eta}{2 \pi|x-\eta|^{2}} \cdot n_{\eta}, \quad x \in \Omega, \eta \in \partial \Omega .
$$

Then $h \in H(\Omega)$ can be expressed as

$$
h(x)=\int_{\partial \Omega} M(x, \eta) \varphi(\eta) d s_{\eta},
$$

where $\varphi$ is the density.

We express the solution as (11); then (25)-(26) implies

$$
\begin{aligned}
\int_{\Omega} \int_{\partial \Omega} M(x, \eta) & \left\{\int_{\mathrm{R}^{2}} K(x-\xi, t) \omega_{0}(\xi) d \xi-\int_{0}^{t} \int_{\Omega} K(x-\xi, t-\tau) F(\xi, \tau) d \xi d \tau\right. \\
& \left.+\int_{0}^{t} \int_{\partial \Omega} K(x-\xi, t-\tau) \rho(\xi, \tau) d s_{\xi} d \tau\right\} \varphi(\eta) d s_{\eta} d x=0,
\end{aligned}
$$

Let

$$
L(\eta, \xi, t)=\int_{\Omega} M(x, \eta) K(x-\xi, t) d x .
$$


Then

$$
\int_{\partial \Omega} \int_{0}^{t} \int_{\partial \Omega} L(\eta, \xi, t-\tau) \rho(\xi, \tau) \varphi(\eta) d s_{\xi} d \tau d s_{\eta}+\int_{\partial \Omega} f(\eta, t) \varphi(\eta) d s_{\eta}=0,
$$

where $f$ is given by (13). Since $\varphi$ is arbitrary, we obtain (12), an integral equation for $\rho$.

There is a variational form of (12). We use the conventional notations of Sobolev spaces $H^{s, \frac{s}{2}}(\Omega \times(0, T))$ or $H^{s, \frac{s}{2}}(\partial \Omega \times(0, T))$ related to parabolic equations,

$$
H^{s, \frac{s}{2}}(\Omega \times(0, T))=L^{2}\left(0, T ; H^{s}(\Omega)\right) \cap H^{\frac{s}{2}}\left(0, T ; L^{2}(\Omega)\right),
$$

equipped with the norm

$$
\|f\|_{s, \frac{s}{2}}=\left(\|f\|_{L^{2}\left(0, T ; H^{s}(\Omega)\right)}^{2}+\|f\|_{H^{\frac{s}{2}}\left(0, T ; L^{2}(\Omega)\right)}^{2}\right)^{\frac{1}{2}} .
$$

As usual, $H_{0}^{s, \frac{s}{2}}(\Omega \times(0, T))$ is the closure of $C_{0}^{\infty}(\Omega \times(0, T])$ with respect to the norm $\|\cdot\|_{s, \frac{s}{2}}$, and $H^{-s,-\frac{s}{2}}(\Omega \times(0, T))$ is defined as the dual space of $H_{0}^{s, \frac{s}{2}}(\Omega \times(0, T))$. Let us define

$$
a(\rho, \varphi)=\int_{0}^{T} \int_{\partial \Omega} \int_{0}^{t} \int_{\partial \Omega} L(\eta, \xi, t-\tau) \rho(\xi, \tau) \varphi(\eta, t) d s_{\xi} d \tau d s_{\eta} d t .
$$

Then (12) can be written as: find $\rho \in H_{0}^{s-2, \frac{s}{2}-1}$, satisfying

$$
a(\rho, \varphi)+\langle f, \varphi\rangle=0, \quad \forall \varphi \in H^{-s,-\frac{s}{2}} .
$$

Since (5)-(9) is well-posed, and (27) is equivalent to it, (27) is also well-posed. However we will use (27) to define approximate solutions, the existence and uniqueness of which is unknown, so we will prove the exixtence and uniqueness of a solution to (27) in the next section.

\section{Approximate solutions}

We will use the generalized Lax-Milgram theorem (see [1]) to study (27). For the reader's convenience we recall it here.

Proposition 3.1. If $H_{1}$ and $H_{2}$ are two real Hilbert spaces, and $a(u, v)$ is a bilinear form on $H_{1} \times H_{2}$ such that

$$
\begin{gathered}
|a(u, v)| \leq C\|u\|_{H_{1}}\|v\|_{H_{2}}, \\
\inf _{u \in H_{1}, u \neq 0} \sup _{v \in H_{2}, v \neq 0} \frac{|a(u, v)|}{\|u\|_{H_{1}}\|v\|_{H_{2}}} \geq C_{0}, \\
\sup _{u \in H_{1}}|a(u, v)|>0, \quad v \neq 0,
\end{gathered}
$$

and if $f \in H_{2}^{\prime}$, then there exists a unique solution to the equation

$$
a(u, v)=f(v), \quad \forall v \in H_{2},
$$

such that

$$
\|u\|_{H_{1}} \leq \frac{1}{C_{0}}\|f\|_{H_{2}^{\prime}} .
$$

We prove the following fact. 
Lemma 3.1. If $s>\frac{3}{2}$ and $f \in H_{0}^{s, \frac{s}{2}}$, then the problem (27) admits a unique solution $\rho \in H_{0}^{s-2, \frac{s}{2}-1}$, and

$$
\|\rho\|_{s-2, \frac{s}{2}-1} \leq C\|f\|_{s, \frac{s}{2}} .
$$

Proof. Let us verify (28). For an arbitrary $\rho \in H_{0}^{s-2, \frac{s}{2}-1}$ we construct a single layer potential

$$
\omega=\int_{0}^{t} \int_{\partial \Omega} K(x-\xi, t-\tau) \rho(\xi, \tau) d s_{\xi} d \tau .
$$

Then it is known that

$$
\|\omega\|_{s-\frac{1}{2}, \frac{s}{2}-\frac{1}{4}} \leq C\|\rho\|_{s-2, \frac{s}{2}-1} .
$$

Let a function $u$ be solved from (6), (7). Then $\nabla \cdot u=0$ and $\omega=-\nabla \wedge u$. From the equation

$$
\frac{\partial \omega}{\partial t}=\triangle \omega
$$

we get

$$
\nabla \wedge\left(\frac{\partial u}{\partial t}-\triangle u\right)=0
$$

so there exists a function $p$ such that

$$
\frac{\partial u}{\partial t}-\triangle u=\nabla p
$$

and hence $u$ is the solution to the Stokes equation.

Let $x \rightarrow \partial \Omega$ in (26); then we get a Fredholm integral equation for $\varphi$ :

$$
h(x)=-\frac{1}{2} \varphi(x)+\int_{\partial \Omega} M(x, \eta) \varphi(\eta) d s_{\eta}, \quad x \in \partial \Omega .
$$

Owing to potential theory the mapping from $h$ to $\varphi$ is an isomorphism in $H^{s}$, where $s$ is a real number. By (24) we have

$$
\int_{\Omega} \omega h d x=\int_{\partial \Omega} u \cdot \nu h d s
$$

We take $h \in H^{-s,-\frac{s}{2}}$ such that

$$
\begin{aligned}
\int_{0}^{T} \int_{\Omega} \omega h d x d t & \geq \frac{1}{2}\|u \cdot \nu\|_{s, \frac{s}{2}}\|h\|_{-s,-\frac{s}{2}} \\
& \geq C\|u \cdot \nu\|_{s, \frac{s}{2}}\|\varphi\|_{-s,-\frac{s}{2}}
\end{aligned}
$$

The estimate for the solutions to the Stokes equation yields

$$
\begin{aligned}
\|\omega\|_{s-\frac{1}{2}, \frac{s}{2}-\frac{1}{4}} & \leq C\|u\|_{s, \frac{s}{2}, \partial \Omega \times(0, T)} \\
& =C\|u \cdot \nu\|_{s, \frac{s}{2}, \partial \Omega \times(0, T)} .
\end{aligned}
$$


There is a one-to-one correspondence between $\rho$ and $\left.\frac{\partial \omega}{\partial n}\right|_{\partial \Omega}$ in $H^{s-2, \frac{s}{2}-1}$, so

$$
\|\rho\|_{s-2, \frac{s}{2}-1} \leq C\|\omega\|_{s-\frac{1}{2}, \frac{s}{2}-\frac{1}{4}}
$$

for $s>2$. (35) is also true for $s \in\left(\frac{3}{2}, 2\right]$. Let us prove this assertion. Let $\varphi \in C_{0}^{\infty}\left(\partial \Omega \times(0, T)\right.$; then we extend it and define $v$ so that $\left.v\right|_{\partial \Omega \times(0, T)}=\varphi$, $v \in C_{0}^{\infty}(\bar{\Omega} \times(0, T))$ and

$$
\|v\|_{-s+\frac{5}{2},-\frac{s}{2}+\frac{5}{4}} \leq C\|\varphi\|_{-s+2,-\frac{s}{2}+1} .
$$

We have

$$
\begin{aligned}
0 & =\int_{0}^{T} \int_{\Omega}\left(\frac{\partial \omega}{\partial t}-\Delta \omega\right) v d x d t \\
& =\int_{0}^{T} \int_{\Omega}\left(\frac{\partial \omega}{\partial t} v+\nabla \omega \cdot \nabla v\right) d x d t-\int_{0}^{T} \int_{\partial \Omega} \frac{\partial \omega}{\partial n} \varphi d s_{x} d t
\end{aligned}
$$

and

$$
\begin{gathered}
\left|\int_{0}^{T} \int_{\Omega} \nabla \omega \cdot \nabla v d x d t\right| \leq C\|v\|_{-s+\frac{5}{2},-\frac{s}{2}+\frac{5}{4}}\|\omega\|_{s-\frac{1}{2}, \frac{s}{2}-\frac{1}{4}} \\
\left|\int_{0}^{T} \int_{\Omega} \frac{\partial \omega}{\partial t} v d x d t\right| \\
\leq C\|v\|_{H^{-\frac{s}{2}+\frac{5}{4}}\left(0, T ; L^{2}(\Omega)\right)}\left\|\frac{\partial \omega}{\partial t}\right\|_{H^{\frac{s}{2}-\frac{5}{4}}\left(0, T ; L^{2}(\Omega)\right)} \\
\leq C\|v\|_{-s+\frac{5}{2},-\frac{s}{2}+\frac{5}{4}}\|\omega\|_{s-\frac{1}{2}, \frac{s}{2}-\frac{1}{4}} .
\end{gathered}
$$

Hence

$$
\left\|\frac{\partial \omega}{\partial n}\right\|_{s-2, \frac{s}{2}-1, \partial \Omega \times(0, T)} \leq C\|\omega\|_{s-\frac{1}{2}, \frac{s}{2}-\frac{1}{4}}
$$

which yields (35).

It follows from (33)-(35) that

$$
\int_{0}^{T} \int_{\Omega} \omega h d x d t \geq C\|\rho\|_{s-2, \frac{s}{2}-1}\|\varphi\|_{-s,-\frac{s}{2}} .
$$

It is easy to see that

$$
a(\rho, \varphi)=\int_{0}^{T} \int_{\Omega} \omega h d x d t
$$

so (28) is verified. To verify the other conditions of Proposition 3.1 is straightforward. 
Using the fundamental solution $K$, the solution $\omega_{k}$ of the scheme (14)-(22) can be expressed as

$$
\begin{aligned}
\omega_{k}\left(x, t_{i}-0\right)= & \int_{\mathrm{R}^{2}} K\left(x-\xi, t_{i}\right) \omega_{0}(\xi) d \xi \\
& -\sum_{j=0}^{i-1} \int_{\Omega} K\left(x-\xi, t_{i}-t_{j}\right) \int_{t_{j}}^{t_{j+1}} F(\xi, \tau) d \xi d \tau \\
& +\sum_{j=0}^{i-1} \int_{\partial \Omega} K\left(x-\xi, t_{i}-t_{j}\right) \int_{t_{j}}^{t_{j+1}} \rho_{k}(\xi, \tau) d \tau d s_{\xi},
\end{aligned}
$$

where $F=\tilde{u}_{k} \cdot \nabla \tilde{\omega}_{k}$ and $\rho_{k}$ is the solution to (18).

\section{UPPER BOUND ESTIMATE FOR LINEAR PROBLEMS}

To analyse the above scheme for the Navier-Stokes equations, we study the same scheme for linear problems first. We replace (5) by

$$
\frac{\partial \omega}{\partial t}+F(x, t)=\triangle \omega,
$$

where $F$ is a known function. This makes (37) (66)-(9) a linear problem. We use the same scheme to solve it, and estimate the approximate solution in this and the next section. Likewise, we assume that the exact solution is sufficiently smooth.

To estimate the approximate solution, let us first define some operators. We define $A=-\triangle+I$, where $I$ is the identity. The semigroup of operators generated from $A$ in $L^{2}\left(\mathrm{R}^{2}\right)$ is denoted by $e^{-A t}$. We define $B: H^{s}(\Omega) \rightarrow H^{s+\frac{1}{2}}(\partial \Omega)$ by $B: \omega \rightarrow g$,

$$
g(\eta)=\int_{\Omega} M(x, \eta) \omega(x) d x,
$$

and the restriction operator from $H^{s}\left(\mathrm{R}^{2}\right)$ to $H^{s}(\Omega)$ is denoted by $R$.

Let us estimate $f_{k}$ first. The first term in (20) is sufficiently regular, so we need to estimate the second term only. Let $i_{0}=\left[\frac{T}{k}\right]$, and

$$
h_{i}=\sum_{j=0}^{i-1} B R e^{-(A-I)\left(t_{i}-t_{j}\right)} \int_{t_{j}}^{t_{j+1}} F(\tau) d \tau,
$$

where we understand $F(\tau)$ to be the zero extension of $F$ to the space $\mathrm{R}^{2}$.

Lemma 4.1. If $2 \leq s<3$, then

$$
\sum_{i=1}^{i_{0}}\left\|h_{i}\right\|_{s, \partial \Omega}^{2} k \leq C\|F\|_{L^{2}\left(0, T ; H^{\beta}(\Omega)\right)}^{2},
$$

where $\frac{1}{2}>\beta>s-\frac{5}{2}$. 
Proof. By the properties of analytic semigroups of operators (see [6]) we have

$$
\begin{aligned}
& \sum_{i=1}^{i_{0}}\left\|h_{i}\right\|_{s, \partial \Omega}^{2} k \leq C \sum_{i=1}^{i_{0}}\left\|\sum_{j=0}^{i-1} e^{-(A-I)\left(t_{i}-t_{j}\right)} \int_{t_{j}}^{t_{j+1}} F(\tau) d \tau\right\|_{s-\frac{1}{2}}^{2} k \\
& \leq C \sum_{i=1}^{i_{0}}\left\|\sum_{j=0}^{i-1} A^{\frac{s}{2}-\frac{1}{4}} e^{-(A-I)\left(t_{i}-t_{j}\right)} \int_{t_{j}}^{t_{j+1}} F(\tau) d \tau\right\|_{0}^{2} k \\
& \leq C \sum_{i=1}^{i_{0}}\left(\sum_{j=0}^{i-1} \int_{t_{j}}^{t_{j+1}}\left(t_{i}-t_{j}\right)^{-\frac{s-\beta}{2}+\frac{1}{4}}\left\|A^{\frac{\beta}{2}} F(\tau)\right\|_{0} d \tau\right)^{2} k \\
& \leq C \sum_{i=1}^{i_{0}}\left(\sum_{j=0}^{i-1} \int_{t_{j}}^{t_{j+1}}\left(t_{i}-t_{j}\right)^{-\frac{s-\beta}{2}+\frac{1}{4}}\|F(\tau)\|_{\beta, \Omega} d \tau\right)^{2} k \\
& \leq C \sum_{i=1}^{i_{0}}\left\{\sum_{j=0}^{i-1} \int_{t_{j}}^{t_{j+1}}\left(t_{i}-t_{j}\right)^{-\frac{s-\beta}{2}+\frac{1}{4}} d \tau\right\} \\
& \left.\leq C \sum_{i=1}^{i_{0}} \sum_{j=0}^{i-1} \int_{t_{j}}^{t_{j+1}}\left(t_{i}-t_{j}\right)^{-\frac{s-\beta}{2}+\frac{1}{4}\|F(\tau)\|_{\beta, \Omega}^{2} d \tau k} \int_{j=0}^{t_{j+1}}\left(t_{i}-t_{j}\right)^{-\frac{s-\beta}{2}+\frac{1}{4}}\|F(\tau)\|_{\beta, \Omega}^{2} d \tau\right\} k \\
& \leq C \sum_{j=0}^{i_{0}-1} \int_{t_{j}}^{t_{j+1}}\|F(\tau)\|_{\beta, \Omega}^{2} d \tau \sum_{i=j+1}^{i_{0}}\left(t_{i}-t_{j}\right)^{-\frac{s-\beta}{2}+\frac{1}{4} k} k \\
& \leq C\|F\|_{L^{2}\left(0, T ; H^{\beta}(\Omega)\right)}^{2} .
\end{aligned}
$$

To estimate $\left\|f_{k}\right\|_{H^{\frac{s}{2}}\left(0, T ; L^{2}(\partial \Omega)\right)}$ we prove one auxiliary lemma first.

Lemma 4.2. If $\beta \in\left(0, \frac{1}{2}\right)$ and $g:\left[0, t_{i_{0}}\right] \rightarrow \mathrm{R}$ is a piecewise constant function, $g(t)=g_{i}, t \in\left(t_{i}, t_{i+1}\right)$, then

$$
\int_{0}^{t_{i_{0}}} \int_{0}^{t_{i_{0}}} \frac{(g(t)-g(\tau))^{2}}{|t-\tau|^{1+2 \beta}} d t d \tau \leq C \sum_{i \neq j} \frac{\left(g_{i}-g_{j}\right)^{2}}{\left|t_{i}-t_{j}\right|^{1+2 \beta}} k^{2} .
$$

Proof. The left hand side can be divided into two parts:

$$
\begin{gathered}
\sum_{i=1}^{i_{0}-1} \int_{t_{i-1}}^{t_{i}} \int_{t_{i}}^{t_{i+1}} \frac{\left(g_{i-1}-g_{i}\right)^{2}}{|t-\tau|^{1+2 \beta}} d t d \tau \leq \frac{k^{1-2 \beta}}{2 \beta(1-2 \beta)} \sum_{i}\left(g_{i+1}-g_{i}\right)^{2}, \\
\sum_{|i-j| \geq 2} \int_{t_{i}}^{t_{i+1}} \int_{t_{j}}^{t_{j+1}} \frac{\left(g_{i}-g_{j}\right)^{2}}{|t-\tau|^{1+2 \beta}} d t d \tau \leq C \sum_{|i-j| \geq 2} \frac{\left(g_{i}-g_{j}\right)^{2}}{\left|t_{i}-t_{j}\right|^{1+2 \beta}} k^{2} .
\end{gathered}
$$

Then the conclusion follows. 
By (19), $\frac{\partial f_{k}}{\partial \tau}$ is a piecewise constant function,

$$
\frac{\partial f_{k}}{\partial \tau}=\frac{1}{k}\left(f_{k i+1}-f_{k i}\right), \quad \tau \in\left(t_{i}, t_{i+1}\right) .
$$

We define

$$
g_{i}=\frac{1}{k}\left(h_{i+1}-h_{i}\right)
$$

Lemma 4.3. If $\frac{5}{2}<s<3$, then

$$
\sum_{i \neq j} \frac{\left\|g_{i}-g_{j}\right\|_{0}^{2}}{\left|t_{i}-t_{j}\right|^{s-1}} k^{2} \leq C\|F\|_{H^{\frac{s}{2}-1}\left(0, T ; H^{-\frac{1}{2}+\varepsilon}(\Omega)\right)}^{2},
$$

where $\varepsilon>0$.

Proof. The left hand side is equal to

$$
\begin{aligned}
& 2 \sum_{j<i} \frac{\left\|g_{i}-g_{j}\right\|_{0}^{2}}{\left|t_{i}-t_{j}\right|^{s-1}} k^{2} \\
& =2 \sum_{j<i} \frac{1}{\left|t_{i}-t_{j}\right|^{s-1}} \|\left(\sum_{l=0}^{i} B R e^{-(A-I)\left(t_{i+1}-t_{l}\right)}\right. \\
& \left.\quad-\sum_{l=0}^{i-1} B R e^{-(A-I)\left(t_{i}-t_{l}\right)}\right) \int_{t_{l}}^{t_{l+1}} F(\tau) d \tau \\
& -\left(\sum_{l=0}^{j} B R e^{-(A-I)\left(t_{j+1}-t_{l}\right)}-\sum_{l=0}^{j-1} B R e^{-(A-I)\left(t_{j}-t_{l}\right)}\right) \int_{t_{l}}^{t_{l+1}} F(\tau) d \tau \|_{0}^{2} .
\end{aligned}
$$

We extend $F$ by zero for $\tau<0$; then the right hand side is equal to

$$
\begin{aligned}
& 2 \sum_{j<i} \frac{1}{\left|t_{i}-t_{j}\right|^{s-1}} \| \sum_{l=j-i}^{j} B R e^{-(A-I)\left(t_{j+1}-t_{l}\right)} \int_{t_{l}}^{t_{l+1}}\left(F\left(\tau+t_{i}-t_{j}\right)-F(\tau)\right) d \tau \\
& -\sum_{l=j-i}^{j-1} B R e^{-(A-I)\left(t_{j}-t_{l}\right)} \int_{t_{l}}^{t_{l+1}}\left(F\left(\tau+t_{i}-t_{j}\right)-F(\tau)\right) d \tau \|_{0}^{2} \\
& \leq 4 \sum_{j<i} \frac{1}{\left|t_{i}-t_{j}\right|^{s-1}} \| \sum_{l=j-i}^{j-1} B R\left(e^{-(A-I)\left(t_{j+1}-t_{l}\right)}\right. \\
& \left.-e^{-(A-I)\left(t_{j}-t_{l}\right)}\right) \int_{t_{l}}^{t_{l+1}}\left(F\left(\tau+t_{i}-t_{j}\right)-F(\tau)\right) d \tau \|_{0}^{2} \\
& +4 \sum_{j<i} \frac{1}{\left|t_{i}-t_{j}\right|^{s-1}}\left\|B R e^{-(A-I) k} \int_{t_{j}}^{t_{j+1}}\left(F\left(\tau+t_{i}-t_{j}\right)-F(\tau)\right) d \tau\right\|_{0}^{2} \\
& \equiv a_{1}+a_{2} \text {. }
\end{aligned}
$$


Due to the properties of analytic semigroups we have

$$
\begin{aligned}
& \left\|B R\left(e^{-(A-I)\left(t_{j+1}-t_{l}\right)}-e^{-(A-I)\left(t_{j}-t_{l}\right)}\right)\left(F\left(\tau+t_{i}-t_{j}\right)-F(\tau)\right)\right\|_{0, \partial \Omega} \\
& \leq C k\left\|A^{\frac{3}{4}} e^{-(A-I)\left(t_{j}-t_{l}\right)}\left(F\left(\tau+t_{i}-t_{j}\right)-F(\tau)\right)\right\|_{0} \\
& \leq C k\left(t_{j}-t_{l}\right)^{-1+\frac{\varepsilon}{2}}\left\|F\left(\tau+t_{i}-t_{j}\right)-F(\tau)\right\|_{-\frac{1}{2}+\varepsilon}
\end{aligned}
$$

then, following the same lines of the proof of Lemma 4.1, we get

$$
a_{1} \leq C \sum_{j<i} \frac{k^{2}}{\left|t_{i}-t_{j}\right|^{s-1}} \sum_{l=j-i}^{j-1} \int_{t_{l}}^{t_{l+1}}\left(t_{j}-t_{l}\right)^{-1+\frac{\varepsilon}{2}}\left\|F\left(\tau+t_{i}-t_{j}\right)-F(\tau)\right\|_{-\frac{1}{2}+\varepsilon}^{2} d \tau .
$$

Let $i^{\prime}=i-j$, then the right hand side is equal to

$$
\begin{aligned}
& C \sum_{i^{\prime}=1}^{i_{0}-1} \sum_{l=-i^{\prime}}^{i_{0}-1-i^{\prime}} \sum_{j>l} \frac{k^{2}}{\left(i^{\prime} k\right)^{s-1}} \int_{t_{l}}^{t_{l+1}}\left(t_{j}-t_{l}\right)^{-1+\frac{\varepsilon}{2}}\left\|F\left(\tau+i^{\prime} k\right)-F(\tau)\right\|_{-\frac{1}{2}+\varepsilon}^{2} d \tau \\
& \leq C \sum_{i^{\prime}=1}^{i_{0}-1} k \sum_{l=-i^{\prime}}^{i_{0}-1-i^{\prime}} \int_{t_{l}}^{t_{l+1}} \frac{\left\|F\left(\tau+i^{\prime} k\right)-F(\tau)\right\|_{-\frac{1}{2}+\varepsilon}^{2}}{\left(i^{\prime} k\right)^{s-1}} d \tau \\
& \leq C\|F\|_{H^{\frac{s}{2}-1}\left(0, T ; H^{-\frac{1}{2}+\varepsilon}(\Omega)\right)}^{2}
\end{aligned}
$$

For the second term,

$$
\begin{aligned}
a_{2} & \leq C \sum_{j<i} \frac{1}{\left|t_{i}-t_{j}\right|^{s-1}}\left(\int_{t_{j}}^{t_{j+1}}\left\|F\left(\tau+t_{i}-t_{j}\right)-F(\tau)\right\|_{-\frac{1}{2}+\varepsilon} d \tau\right)^{2} \\
& \leq C \sum_{i=1}^{i_{0}} \sum_{j<i} \frac{k \int_{t_{j}}^{t_{j+1}}\left\|F\left(\tau+t_{i}-t_{j}\right)-F(\tau)\right\|_{-\frac{1}{2}+\varepsilon}^{2} d \tau}{\left|t_{i}-t_{j}\right|^{s-1}} \\
& =C \sum_{i^{\prime}=1}^{i_{0}-1} \sum_{j=0}^{i_{0}-1-i^{\prime}} \frac{k \int_{t_{j}}^{t_{j+1}}\left\|F\left(\tau+i^{\prime} k\right)-F(\tau)\right\|_{-\frac{1}{2}+\varepsilon}^{2} d \tau}{\left(i^{\prime} k\right)^{s-1}} \\
& \leq C\|F\|_{H^{\frac{s}{2}-1}\left(0, T ; H^{-\frac{1}{2}+\varepsilon}(\Omega)\right)}^{2} .
\end{aligned}
$$

Thus the proof is complete.

Combining Lemma 4.1 Lemma 4.2, and Lemma 4.3 we get for $\frac{5}{2}<s<3$ that

$$
\left\|f_{k}\right\|_{s, \frac{s}{2}} \leq C\left\{\|F\|_{L^{2}\left(0, T ; H^{\beta}(\Omega)\right)}+\|F\|_{H^{\frac{s}{2}-1}\left(0, T ; H^{-\frac{1}{2}+\varepsilon}(\Omega)\right)}\right\}+C\left(\omega_{0}\right),
$$

where $C\left(\omega_{0}\right)$ is a constant depending on $\omega_{0}$ only. Lemma 3.1 implies

$$
\left\|\rho_{k}\right\|_{s-2, \frac{s}{2}-1} \leq C\left\{\|F\|_{L^{2}\left(0, T ; H^{\beta}(\Omega)\right)}+\|F\|_{H^{\frac{s}{2}-1}\left(0, T ; H^{-\frac{1}{2}+\varepsilon}(\Omega)\right)}\right\}+C\left(\omega_{0}\right) .
$$

We are going to estimate the solutions $\omega_{k}$ and $\tilde{\omega}_{k}$. Equation (14) is reduced to

$$
\frac{\partial \tilde{\omega}_{k}}{\partial t}+F=0
$$

hence

$$
\tilde{\omega}_{k}(\tau)=-\int_{t_{i}}^{\tau} F d \tau+\omega_{k}\left(t_{i}-0\right)
$$


so $\tilde{\omega}_{k}$ can be estimated in terms of $F$ and $\omega_{k}$. It suffices to estimate $\omega_{k}$. We define a vector function $\chi_{k}$ satisfying

$$
\begin{gathered}
\frac{\partial \chi_{k}}{\partial t}=\triangle \chi_{k}, \\
\left.\chi_{k}\right|_{x \in \partial \Omega}=\rho_{k} n, \\
\left.\chi_{k}\right|_{t=0}=0 .
\end{gathered}
$$

Lemma 4.4. We have

$$
\left\|\chi_{k}\right\|_{s-\frac{3}{2}, \frac{s}{2}-\frac{3}{4}, \Omega \times(0, T)} \leq C\left\|\rho_{k}\right\|_{s-2, \frac{s}{2}-1, \partial \Omega \times(0, T)}
$$

for all $s \in \mathrm{R}$, provided $\rho_{k} \in H_{0}^{s-2, \frac{s}{2}-1}$ and $\chi_{k}$ is a classical solution.

Proof. By classical potential theory, (41) is valid for $s>2$. If $s<-2$, we take $\varphi \in C_{0}^{\infty}(\Omega \times(0, T))$ and let $v$ satisfy

$$
\begin{gathered}
\frac{\partial v}{\partial t}+\Delta v=\varphi \\
\left.v\right|_{x \in \partial \Omega}=0 \\
\left.v\right|_{t=T}=0 .
\end{gathered}
$$

We have

$$
\begin{aligned}
& \int_{0}^{T} \int_{\Omega} \chi_{k} \varphi d x d t=\int_{0}^{T} \int_{\Omega} \chi_{k}\left(\frac{\partial v}{\partial t}+\Delta v\right) d x d t \\
= & \int_{0}^{T} \int_{\Omega}\left(-v \frac{\partial \chi_{k}}{\partial t}+v \Delta \chi_{k}\right) d x d t+\int_{0}^{T} \int_{\partial \Omega} \chi_{k} \frac{\partial v}{\partial n} d s_{x} d t \\
= & \int_{0}^{T} \int_{\partial \Omega} \rho_{k} n \frac{\partial v}{\partial n} d s_{x} d t
\end{aligned}
$$

Then

$$
\begin{aligned}
\left|\int_{0}^{T} \int_{\Omega} \chi_{k} \varphi d x d t\right| & \leq C\left\|\rho_{k}\right\|_{s-2, \frac{s}{2}-1}\|v\|_{3-s, \frac{3}{2}-\frac{s}{2}} \\
& \leq C\left\|\rho_{k}\right\|_{s-2, \frac{s}{2}-1}\|\varphi\|_{\frac{3}{2}-s, \frac{3}{4}-\frac{s}{2}}
\end{aligned}
$$

which yields (41). For $s \in[-2,2]$, (41) can be obtained by interpolation.

Theorem 4.1. If $1<\sigma<\frac{3}{2}$, then

$$
\left\|\omega_{k}\left(t_{i}-0\right)\right\|_{\sigma, \Omega} \leq C\|F\|_{L^{2}\left(0, T ; H^{s-\frac{5}{2}}(\Omega)\right)}+C\left\|\rho_{k}\right\|_{s-2, \frac{s}{2}-1}+C\left(\omega_{0}\right),
$$

for all $i$, where $\sigma+\frac{3}{2}<s<3$.

Proof. We apply (36). Let us consider the third term first. By integration by parts, we have

$$
\begin{aligned}
& \int_{\partial \Omega} K\left(x-\xi, t_{i}-t_{j}\right) \rho_{k}(\xi, \tau) d s_{\xi} \\
= & \int_{\Omega} \nabla_{\xi} K\left(x-\xi, t_{i}-t_{j}\right) \cdot \chi_{k} d \xi+\int_{\Omega} K\left(x-\xi, t_{i}-t_{j}\right) \nabla_{\xi} \cdot \chi_{k} d \xi .
\end{aligned}
$$


Let $\chi_{k}$ be extended by zero to $\mathrm{R}^{2}$. In view of Lemma 4.4 we get

$$
\begin{aligned}
& \left\|\sum_{j=0}^{i-1} R A^{\frac{1}{2}} e^{-(A-I)\left(t_{i}-t_{j}\right)} \int_{t_{j}}^{t_{j+1}} \chi_{k}(\tau) d \tau\right\|_{\sigma} \\
& \leq C \sum_{j=0}^{i-1} \int_{t_{j}}^{t_{j+1}}\left\|A^{\frac{1+\sigma}{2}} e^{-(A-I)\left(t_{i}-t_{j}\right)} \chi_{k}(\tau)\right\|_{0} d \tau \equiv a_{1} .
\end{aligned}
$$

We take $r=s-\frac{5}{2} \in\left(\sigma-1, \frac{1}{2}\right)$; then

$$
\begin{aligned}
a_{1} & \leq C \sum_{j=0}^{i-1} \int_{t_{j}}^{t_{j+1}}\left(t_{i}-t_{j}\right)^{-\frac{1+\sigma-r}{2}}\left\|\chi_{k}(\tau)\right\|_{r} d \tau \\
& \leq C\left(\sum_{j=0}^{i-1} \int_{t_{j}}^{t_{j+1}}\left(t_{i}-t_{j}\right)^{-\frac{1+\sigma-r}{2} p} d \tau\right)^{\frac{1}{p}}\left(\sum_{j=0}^{i-1} \int_{t_{j}}^{t_{j+1}}\left\|\chi_{k}(\tau)\right\|_{r}^{q} d \tau\right)^{\frac{1}{q}},
\end{aligned}
$$

where $\frac{1}{p}+\frac{1}{q}=1$. We take $p=\frac{4}{2 s-1-2 r}$; then $\frac{1+\sigma-r}{2} p<1$, and due to the interpolation of Sobolev spaces we get

$$
a_{1} \leq C\left\|\chi_{k}\right\|_{L^{q}\left(0, T ; H^{r}(\Omega)\right)} \leq C\left\|\chi_{k}\right\|_{s-\frac{3}{2}, \frac{s}{2}-\frac{3}{4}} .
$$

For the second term of (42) we extend $\nabla \cdot \chi_{k}$ by zero to $\mathrm{R}^{2}$ and note that $\left\|\nabla \cdot \chi_{k}\right\|_{r, \mathrm{R}^{2}} \leq C\left\|\chi_{k}\right\|_{1+r \Omega \Omega}$, then take $p=q=2$ to obtain

$$
\begin{aligned}
& \left\|\sum_{j=0}^{i-1} R e^{-(A-I)\left(t_{i}-t_{j}\right)} \int_{t_{j}}^{t_{j+1}} \nabla \cdot \chi_{k}(\tau) d \tau\right\|_{\sigma} \\
& \leq C \sum_{j=0}^{i-1} \int_{t_{j}}^{t_{j+1}}\left(t_{i}-t_{j}\right)^{-\frac{\sigma-r}{2}}\left\|\chi_{k}(\tau)\right\|_{1+r} d \tau \\
& \leq C\left(\sum_{j=0}^{i-1} \int_{t_{j}}^{t_{j+1}}\left(t_{i}-t_{j}\right)^{-\sigma+r} d \tau\right)^{\frac{1}{2}}\left(\sum_{j=0}^{i-1} \int_{t_{j}}^{t_{j+1}}\left\|\chi_{k}(\tau)\right\|_{1+r}^{2} d \tau\right)^{\frac{1}{2}} \\
& \leq C\left\|\chi_{k}\right\|_{L^{2}\left(0, T ; H^{s-\frac{3}{2}}\right)} .
\end{aligned}
$$

The second term of (36) can be estimated in the same way.

\section{ERror estimate For LineAR PROBlems}

Lemma 5.1. If $0<\beta<\frac{1}{2}$ and $\varepsilon>0$, then

$$
\left\|f-f_{k}\right\|_{\frac{3}{2}+\varepsilon, \frac{3}{4}+\frac{\varepsilon}{2}} \leq C k^{\frac{7}{16}+\frac{3 \beta}{8}-\frac{\varepsilon}{2}} .
$$

Proof. We get from (13) and (20) that

$$
f\left(t_{i}\right)-f_{k i}=\sum_{j=0}^{i-1} \int_{t_{j}}^{t_{j+1}} B R\left(e^{-(A-I)\left(t_{i}-\tau\right)}-e^{-(A-I)\left(t_{i}-t_{j}\right)}\right) F(\tau) d \tau .
$$


Let $G(\tau)=\left(I-e^{-(A-I)\left(\tau-t_{j}\right)}\right) F(\tau)$ and

$$
\begin{gathered}
a_{1}=\sum_{j=0}^{i-2} \int_{t_{j}}^{t_{j+1}} B R e^{-(A-I)\left(t_{i}-\tau\right)} G(\tau) d \tau \\
a_{2}=\int_{t_{i-1}}^{t_{i}} B R e^{-(A-I)\left(t_{i}-\tau\right)} G(\tau) d \tau
\end{gathered}
$$

then $f\left(t_{i}\right)-f_{k i}=a_{1}+a_{2}$. We have

$$
\begin{aligned}
\left\|a_{1}\right\|_{\frac{3}{2}+\varepsilon, \partial \Omega} & \leq C\left\|a_{1}\right\|_{2+\varepsilon, \Omega} \leq C \sum_{j=0}^{i-2} \int_{t_{j}}^{t_{j+1}}\left\|e^{-(A-I)\left(t_{i}-\tau\right)} G(\tau)\right\|_{1+\varepsilon} d \tau \\
& \leq C \sum_{j=0}^{i-2} \int_{t_{j}}^{t_{j+1}}\left(t_{i}-\tau\right)^{-1+\frac{\varepsilon}{2}}\|G(\tau)\|_{-1+2 \varepsilon} d \tau \\
& \leq C \sum_{j=0}^{i-2} \int_{t_{j}}^{t_{j+1}}\left(t_{i}-\tau\right)^{-1+\frac{\varepsilon}{2}} k^{\frac{1+\beta}{2}-\varepsilon}\|F(\tau)\|_{\beta} d \tau
\end{aligned}
$$

and therefore

$$
\begin{aligned}
\sum_{i=1}^{i_{0}} \| & a_{1} \|_{\frac{3}{2}+\varepsilon, \partial \Omega}^{2} k \leq C \sum_{i=1}^{i_{0}}\left(\sum_{j=0}^{i-2} \int_{t_{j}}^{t_{j+1}}\left(t_{i}-\tau\right)^{-1+\frac{\varepsilon}{2}} k^{\frac{1+\beta}{2}-\varepsilon}\|F(\tau)\|_{\beta} d \tau\right)^{2} k \\
\leq & C \sum_{i=1}^{i_{0}}\left(\sum_{j=0}^{i-2} \int_{t_{j}}^{t_{j+1}}\left(t_{i}-\tau\right)^{-1+\frac{\varepsilon}{2}} d \tau\right) \\
& \cdot\left(\sum_{j=0}^{i-2} \int_{t_{j}}^{t_{j+1}}\left(t_{i}-\tau\right)^{-1+\frac{\varepsilon}{2}} k^{1+\beta-2 \varepsilon}\|F(\tau)\|_{\beta}^{2} d \tau\right) k \\
\leq & C \sum_{j=0}^{i_{0}-2} \int_{t_{j}}^{t_{j+1}}\|F(\tau)\|_{\beta}^{2} k^{1+\beta-2 \varepsilon} \sum_{i=j+2}^{i_{0}}\left(t_{i}-\tau\right)^{-1+\varepsilon} k d \tau \\
\leq & C k^{1+\beta-2 \varepsilon}\|F\|_{L^{2}\left(0, T ; H^{\beta}(\Omega)\right)}^{2} .
\end{aligned}
$$

Besides, we have

$$
\begin{aligned}
\sum_{i=1}^{i_{0}} \| & a_{2} \|_{\frac{3}{2}+\varepsilon, \partial \Omega}^{2} k \\
& \leq \sum_{i=1}^{i_{0}}\left(\int_{t_{i-1}}^{t_{i}}\left\|e^{-(A-I)\left(t_{i}-\tau\right)}\left(I-e^{-(A-I)\left(\tau-t_{i-1}\right)}\right) F(\tau)\right\|_{1+\varepsilon} d \tau\right)^{2} k \\
& \leq C \sum_{i=1}^{i_{0}} \int_{t_{i-1}}^{t_{i}}\|F(\tau)\|_{\beta}^{2} d \tau \int_{t_{i-1}}^{t_{i}}\left(t_{i}-\tau\right)^{-1-\varepsilon+\beta} k d \tau \\
& \leq C k^{1+\beta-\varepsilon}\|F\|_{L^{2}\left(0, T ; H^{\beta}(\Omega)\right)}^{2} .
\end{aligned}
$$


Consequently,

$$
\sum_{i=1}^{i_{0}}\left\|f\left(t_{i}\right)-f_{k i}\right\|_{\frac{3}{2}+\varepsilon, \partial \Omega}^{2} k \leq C k^{1+\beta-2 \varepsilon}\|F\|_{L^{2}\left(0, T ; H^{\beta}(\Omega)\right)}^{2} .
$$

In the same way,

$$
\sum_{i=1}^{i_{0}}\left\|f\left(t_{i}\right)-f_{k i}\right\|_{0, \partial \Omega}^{2} k \leq C k^{2}\|F\|_{L^{2}\left(0, T ; H^{\beta}(\Omega)\right)}^{2} .
$$

Let

$$
\begin{aligned}
h_{i} & =\frac{1}{k}\left(f\left(t_{i}\right)-f_{k i}-f\left(t_{i-1}\right)+f_{k i-1}\right) \\
& =\frac{1}{k} \sum_{j=0}^{i-2} \int_{t_{j}}^{t_{j+1}} B R\left(e^{-(A-I)\left(t_{i}-\tau\right)}-e^{-(A-I)\left(t_{i-1}-\tau\right)}\right) G(\tau) d \tau+\frac{a_{2}}{k} .
\end{aligned}
$$

Following the same lines, we can prove that

$$
\sum_{i=1}^{i_{0}}\left\|h_{i}\right\|_{0}^{2} k \leq C k^{\frac{1}{2}+\beta}\|F\|_{L^{2}\left(0, T ; H^{\beta}(\Omega)\right)}^{2} .
$$

The function $f_{k}$ is linear in each interval $\left[t_{i-1}, t_{i}\right]$. The function $f$ is not linear, but $f$ is sufficiently smooth. It is easy to estimate the difference between $f$ and its linear interpolation; then by (44)-(46) we get

$$
\begin{gathered}
\left\|f-f_{k}\right\|_{L^{2}\left(0, T ; H^{\frac{3}{2}+\varepsilon}(\partial \Omega)\right)} \leq C k^{\frac{1+\beta}{2}-\varepsilon}, \\
\left\|f-f_{k}\right\|_{L^{2}\left(0, T ; L^{2}(\partial \Omega)\right)} \leq C k \\
\left\|f-f_{k}\right\|_{H^{1}\left(0, T ; L^{2}(\partial \Omega)\right)} \leq C k^{\frac{1}{4}+\frac{\beta}{2}} .
\end{gathered}
$$

By interpolation we get

$$
\left\|f-f_{k}\right\|_{H^{\frac{3}{4}+\frac{\varepsilon}{2}}\left(0, T ; L^{2}(\partial \Omega)\right)} \leq C k^{\frac{7}{16}+\frac{3 \beta}{8}-\frac{\varepsilon}{2}} .
$$

Then (43) follows.

Lemma 5.1 and Lemma 3.1 imply

$$
\left\|\rho-\rho_{k}\right\|_{-\frac{1}{2}+\varepsilon,-\frac{1}{4}+\frac{\varepsilon}{2}} \leq C k^{\frac{7}{16}+\frac{3 \beta}{8}-\frac{\varepsilon}{2}} .
$$

We turn now to estimating the error $\omega-\omega_{k}$. 
Theorem 5.1. If $1<\sigma<\frac{3}{2}$, then

$$
\left\|\omega\left(t_{i}\right)-\omega_{k}\left(t_{i}-0\right)\right\|_{\sigma-2, \Omega} \leq C k^{\frac{7}{16}+\frac{3 \beta}{8}-\frac{\varepsilon}{2}},
$$

for all $i$, where $0<\beta<\frac{1}{2}, \varepsilon>0$.

Proof. We get from (11) and (36) that

$$
\begin{aligned}
\omega\left(x, t_{i}\right) & -\omega_{k}\left(x, t_{i}-0\right) \\
= & -\sum_{j=0}^{i-1} \int_{t_{j}}^{t_{j+1}} \int_{\Omega}\left(K\left(x-\xi, t_{i}-\tau\right)-K\left(x-\xi, t_{i}-t_{j}\right)\right) F(\xi, \tau) d \xi d \tau \\
& +\sum_{j=0}^{i-1} \int_{t_{j}}^{t_{j+1}} \int_{\partial \Omega}\left(K\left(x-\xi, t_{i}-\tau\right) \rho(\xi, \tau)-K\left(x-\xi, t_{i}-t_{j}\right) \rho_{k}(\xi, \tau)\right) d s_{\xi} d \tau \\
= & -\sum_{j=0}^{i-1} \int_{t_{j}}^{t_{j+1}} \int_{\Omega}\left(K\left(x-\xi, t_{i}-\tau\right)-K\left(x-\xi, t_{i}-t_{j}\right)\right) F(\xi, \tau) d \xi d \tau \\
& +\sum_{j=0}^{i-1} \int_{t_{j}}^{t_{j+1}} \int_{\partial \Omega}\left(K\left(x-\xi, t_{i}-\tau\right)-K\left(x-\xi, t_{i}-t_{j}\right)\right) \rho_{k}(\xi, \tau) d s_{\xi} d \tau \\
& +\sum_{j=0}^{i-1} \int_{t_{j}}^{t_{j+1}} \int_{\partial \Omega} K\left(x-\xi, t_{i}-\tau\right)\left(\rho(\xi, \tau)-\rho_{k}(\xi, \tau)\right) d s_{\xi} d \tau \\
\equiv & a_{1}+a_{2}+a_{3} .
\end{aligned}
$$

The sum in $a_{1}, a_{2}$ can be divided into two parts: $j=0, \cdots, i-2$, and $j=i-1$. For $j=i-1$ we have

$$
\begin{gathered}
\left\|\int_{t_{i-1}}^{t_{i}}\left(e^{-(A-I)\left(t_{i}-\tau\right)}-e^{-(A-I) k}\right) F(\tau) d \tau\right\|_{\sigma-2} \\
\quad \leq C \int_{t_{i-1}}^{t_{i}}\|F(\tau)\|_{\beta} d \tau \leq C k,
\end{gathered}
$$

and for $j=0, \cdots, i-2$ we have

$$
\begin{gathered}
\left\|\sum_{j=0}^{i-2} \int_{t_{j}}^{t_{j+1}}\left(e^{-(A-I)\left(t_{i}-\tau\right)}-e^{-(A-I)\left(t_{i}-t_{j}\right)}\right) F(\tau) d \tau\right\|_{\sigma-2} \\
\quad \leq C \sum_{j=0}^{i-2} \int_{t_{j}}^{t_{j+1}}\left\|\int_{t_{i}-t_{j}}^{t_{i}-\tau} e^{-(A-I) \zeta} d \zeta F(\tau)\right\|_{\sigma} d \tau \\
\quad \leq C k \sum_{j=0}^{i-2} \int_{t_{j}}^{t_{j+1}}\left(t_{i}-\tau\right)^{-\frac{\sigma-\beta}{2}}\|F(\tau)\|_{\beta} d \tau \leq C k .
\end{gathered}
$$


We get for the first part of $a_{2}$ that

$$
\begin{aligned}
\left\|a_{21}\right\|_{\sigma-2}= & \| \int_{t_{i-1}}^{t_{i}} R A^{\frac{1}{2}}\left(e^{-(A-I)\left(t_{i}-\tau\right)}-e^{-(A-I) k}\right) \chi_{k}(\tau) d \tau \\
& +\int_{t_{i-1}}^{t_{i}} R\left(e^{-(A-I)\left(t_{i}-\tau\right)}-e^{-(A-I) k}\right) \nabla \cdot \chi_{k}(\tau) d \tau \|_{\sigma-2} \\
\leq & C \int_{t_{i-1}}^{t_{i}}\left\|A^{\frac{\sigma}{2}-\frac{1}{2}}\left(e^{-(A-I)\left(t_{i}-\tau\right)}-e^{-(A-I) k}\right) \chi_{k}(\tau)\right\|_{0} d \tau \\
& +C \int_{t_{i-1}}^{t_{i}}\left\|A^{\frac{\sigma}{2}-1}\left(e^{-(A-I)\left(t_{i}-\tau\right)}-e^{-(A-I) k}\right) \nabla \cdot \chi_{k}(\tau)\right\|_{0} d \tau \\
\leq & C \int_{t_{i-1}}^{t_{i}}\left(t_{i}-\tau\right)^{\frac{s-\sigma}{2}-\frac{3}{4}-\frac{\varepsilon}{2}}\left\|\chi_{k}(\tau)\right\|_{s-\frac{5}{2}-\varepsilon} d \tau \\
& +C \int_{t_{i-1}}^{t_{i}}\left(t_{i}-\tau\right)^{\frac{s-\sigma}{2}-\frac{1}{4}}\left\|\chi_{k}(\tau)\right\|_{s-\frac{3}{2}} d \tau \\
\leq & C \int_{t_{i-1}}^{t_{i}}\left(t_{i}-\tau\right)^{\frac{s-\sigma}{2}-\frac{3}{4}-\frac{\varepsilon}{2}} d \tau \sup _{\tau}\left\|_{k}(\tau)\right\|_{s-\frac{5}{2}-\varepsilon} \\
& +C\left(\int_{t_{i-1}}^{t_{i}}\left(t_{i}-\tau\right)^{s-\sigma-\frac{1}{2}} d \tau\right)^{\frac{1}{2}}\left(\int_{t_{i-1}}^{t_{i}}\left\|\chi_{k}(\tau)\right\|_{s-\frac{3}{2}}^{2} d \tau\right)^{\frac{1}{2}} .
\end{aligned}
$$

We take $s=\sigma+\frac{3}{2}$ and notice (41), then get

$$
\left\|a_{21}\right\|_{\sigma-2} \leq C k^{1-\frac{\varepsilon}{2}} .
$$

For the second part of $a_{2}$ we can repeat the proof of Theorem 4.1 and get an upper bound of $C k$. The details are omitted here.

Finally, by potential theory and (47) we have

$$
\left\|a_{3}\right\|_{1+\varepsilon, \frac{1}{2}+\frac{\varepsilon}{2}} \leq C k^{\frac{7}{16}+\frac{3 \beta}{8}-\frac{\varepsilon}{2}} .
$$

Then by the embedding theorem we get

$$
\left\|a_{3}\right\|_{0, \Omega} \leq C k^{\frac{7}{16}+\frac{3 \beta}{8}-\frac{\varepsilon}{2}} .
$$

Combining the above results, we obtain (48).

\section{Estimates for the NAVier-Stokes Equation}

For the Navier-Stokes equation we denote $F_{0}=u \cdot \nabla \omega$, where $u$ and $\omega$ are exact solutions; then we regard $F_{0}$ as a known function. We denote by $f^{*}, \rho^{*}$ and $\omega^{*}$ the approximate solutions to

$$
\frac{\partial \omega}{\partial t}+F_{0}=\triangle \omega
$$

and (6) -(92). Then we define

$$
\tilde{\omega}^{*}(\tau)=-\int_{t_{i}}^{\tau} F_{0} d \tau+\omega^{*}\left(t_{i}-0\right), \quad \tau \in\left[t_{i}, t_{i+1}\right) .
$$


The estimates in Section 4 and Section 5 are valid for $\omega^{*}$ and $\tilde{\omega}^{*}$, namely, we have

$$
\begin{gathered}
\left\|\omega^{*}\left(t_{i}-0\right)\right\|_{\sigma},\left\|\tilde{\omega}^{*}(t)\right\|_{\sigma} \leq C_{1}, \\
\left\|\omega\left(t_{i}\right)-\omega^{*}\left(t_{i}-0\right)\right\|_{\sigma-2, \Omega} \leq C_{2} k^{\frac{7}{16}+\frac{3 \beta}{8}-\frac{\varepsilon}{2}}
\end{gathered}
$$

for $0 \leq i \leq i_{0}$ and $0 \leq t \leq T$. Certainly the constants $C_{1}, C_{2}$ depend on the exact solution. Using this auxiliary solution we estimate $\omega^{*}-\omega_{k}$; then we obtain the estimates for $\omega-\omega_{k}$.

We need one fact about Sobolev spaces. For the reader's convenience we recall it here:

Lemma 6.1. If $h, g \in H^{r}(\Omega)$ with $1<r<2$, then

$$
\|h g\|_{r} \leq C\|h\|_{r}\|g\|_{r},
$$

and if $h \in H^{1+\varepsilon}(\Omega), g \in H^{r}(\Omega)$ with $\varepsilon>0,0 \leq r \leq 1$, then

$$
\|h g\|_{r} \leq C\|h\|_{1+\varepsilon}\|g\|_{r} .
$$

To estimate the upper bound and error for non-linear problems, let us prove the following lemmas first.

Lemma 6.2. Let $g_{i}$ be defined by (38). Then

$$
\sum_{i=1}^{i_{0}}\left\|g_{i}\right\|_{0, \partial \Omega}^{2} k \leq C\|F\|_{L^{2}\left(0, T ; H^{-\frac{1}{2}+\varepsilon}(\Omega)\right)}^{2},
$$

where $\varepsilon>0$.

Proof. We notice that

$$
\begin{aligned}
g_{i}= & \frac{1}{k} \sum_{j=0}^{i-1} B R\left(e^{-(A-I)\left(t_{i+1}-t_{j}\right)}-e^{-(A-I)\left(t_{i}-t_{j}\right)}\right) \int_{t_{j}}^{t_{j+1}} F(\tau) d \tau \\
& +\frac{1}{k} B R e^{-(A-I) k} \int_{t_{i}}^{t_{i+1}} F(\tau) d \tau \\
= & -\sum_{j=0}^{i-1} B R \frac{1}{k} \int_{t_{i}-t_{j}}^{t_{i+1}-t_{j}}(A-I) e^{-(A-I) \zeta} d \zeta \int_{t_{j}}^{t_{j+1}} F(\tau) d \tau \\
& +B R e^{-(A-I) k} \frac{1}{k} \int_{t_{i}}^{t_{i+1}} F(\tau) d \tau .
\end{aligned}
$$

Following the same lines as the proof of Lemma 4.1, we get (154).

For the Navier-Stokes equation, $F=\tilde{u}_{k} \cdot \nabla \tilde{\omega}_{k}$. We apply Lemma 4.1 and Lemma 6.2 to obtain

$$
\left\|f_{k}\right\|_{2,1} \leq C\|F\|_{L^{2}\left(0, T ; H^{-\frac{1}{2}+\varepsilon}(\Omega)\right)}+C\left(\omega_{0}\right) ;
$$

then Lemma 3.1 gives

$$
\left\|\rho_{k}\right\|_{0,0} \leq C\|F\|_{L^{2}\left(0, T ; H^{-\frac{1}{2}+\varepsilon}(\Omega)\right)}+C\left(\omega_{0}\right) .
$$

Lemma 6.3.

$$
\left\|\tilde{u}_{k} \cdot \nabla \tilde{\omega}_{k}\right\|_{-\frac{1}{2}+\varepsilon} \leq C\left\|\tilde{\omega}_{k}\right\|_{\frac{1}{2}+\varepsilon}^{2}
$$


Proof. We have

$$
\tilde{u}_{k} \cdot \nabla \tilde{\omega}_{k}=-\nabla \wedge\left(\left(\tilde{u}_{k} \cdot \nabla\right) \tilde{u}_{k}\right)=-\nabla \wedge\left(\nabla\left(\tilde{u}_{k} \otimes \tilde{u}_{k}\right)\right) .
$$

Lemma 6.1 implies

$$
\left\|\tilde{u}_{k} \cdot \nabla \tilde{\omega}_{k}\right\|_{-\frac{1}{2}+\varepsilon}=\left\|\tilde{u}_{k} \otimes \tilde{u}_{k}\right\|_{\frac{3}{2}+\varepsilon} \leq C\left\|\tilde{u}_{k}\right\|_{\frac{3}{2}+\varepsilon}^{2} \leq C\left\|\tilde{\omega}_{k}\right\|_{\frac{1}{2}+\varepsilon}^{2} \cdot
$$

Lemma 6.4. Assume that $1<\sigma<\frac{3}{2}, 0<\varepsilon \leq \frac{\sigma-1}{2}$, and

$$
\left\|\tilde{\omega}_{k}\right\|_{L^{2}\left(0, T ; H^{\sigma-\varepsilon}(\Omega)\right)}+\left\|\tilde{\omega}_{k}\right\|_{\sigma-1-\varepsilon} \leq M_{0} .
$$

Then

$$
\left(\sum_{l=0}^{i}\left\|\omega_{k}\left(t_{l}-0\right)\right\|_{\sigma}^{2} k\right)^{\frac{1}{2}}+\left\|\omega_{k}\left(t_{i}-0\right)\right\|_{\sigma-1}+k\left\|\omega_{k}\left(t_{i}-0\right)\right\|_{\sigma+1} \leq M_{1},
$$

where $M_{1}$ depends on $M_{0}$.

Proof. We apply (36) and (42) to estimate $\omega_{k}\left(t_{i}-0\right)$. We take $\beta \in\left(\sigma-1, \frac{1}{2}\right)$, then get

$$
\left\|\sum_{j=0}^{i-1} \int_{t_{j}}^{t_{j+1}} A^{\frac{1}{2}} e^{-(A-I)\left(t_{i}-t_{j}\right)} \chi_{k}(\tau) d \tau\right\|_{\sigma} \leq C \sum_{j=0}^{i-1} \int_{t_{j}}^{t_{j+1}}\left(t_{i}-t_{j}\right)^{-\frac{1+\sigma-\beta}{2}}\left\|\chi_{k}(\tau)\right\|_{\beta} d \tau .
$$

Then

$$
\begin{gathered}
\sum_{i}\left\|\sum_{j=0}^{i-1} \int_{t_{j}}^{t_{j+1}} A^{\frac{1}{2}} e^{-(A-I)\left(t_{i}-t_{j}\right)} \chi_{k}(\tau) d \tau\right\|_{\sigma}^{2} k \\
\leq C \int_{0}^{T}\left\|\chi_{k}(\tau)\right\|_{\beta}^{2} d \tau=C\left\|\chi_{k}\right\|_{L^{2}\left(0, T ; H^{\beta}(\Omega)\right)}^{2} .
\end{gathered}
$$

The other terms in (36) and (42) can be estimated in the same way. By Lemma 4.4 .

$$
\left\|\chi_{k}\right\|_{L^{2}\left(0, T ; H^{\beta}(\Omega)\right)} \leq C\left\|\rho_{k}\right\|_{\beta-\frac{1}{2}, \frac{\beta}{2}-\frac{1}{4}} .
$$

Now (56) can be applied. We obtain

$$
\sum_{l}\left\|\omega_{k}\left(t_{l}-0\right)\right\|_{\sigma}^{2} k \leq C\|F\|_{L^{2}\left(0, T ; H^{-\frac{1}{2}+\varepsilon}(\Omega)\right)}^{2}+C\left(\omega_{0}\right) .
$$

Due to Lemma 6.3,

$$
\sum_{l}\left\|\omega_{k}\left(t_{l}-0\right)\right\|_{\sigma}^{2} k \leq C\left\|\tilde{\omega}_{k}\right\|_{L^{4}\left(0, T ; H^{\frac{1}{2}+\varepsilon}(\Omega)\right)}^{4}+C\left(\omega_{0}\right) .
$$

We apply the interpolation inequality to obtain

$$
\int_{0}^{T}\left\|\tilde{\omega}_{k}\right\|_{\frac{1}{2}+\varepsilon}^{4} d t \leq \int_{0}^{T}\left\|\tilde{\omega}_{k}\right\|_{\sigma-\varepsilon}^{6-4 \sigma+8 \varepsilon}\left\|\tilde{\omega}_{k}\right\|_{\sigma-1-\varepsilon}^{4 \sigma-2-8 \varepsilon} d t .
$$

We notice that $6-4 \sigma+8 \varepsilon \leq 2$, so the assumption (57) implies the right hand side is bounded by a constant depending on $M_{0}$. Following the proof of Theorem 4.1, the upper bounds for $\left\|\tilde{\omega}_{k}\left(t_{i}-0\right)\right\|_{\sigma-1}$ and $k\left\|\tilde{\omega}_{k}\left(t_{i}-0\right)\right\|_{\sigma+1}$ can also be obtained. 
Lemma 6.5. If (58) holds for $i \leq i_{1}$, then there exists a constant $k_{0}>0$, depending on $M_{1}$, such that if $k \leq k_{0}$, then

$$
\left\|\tilde{\omega}_{k}(\tau)\right\|_{r} \leq C_{3}\left\|\omega_{k}\left(t_{i}-0\right)\right\|_{r}, \quad t_{i} \leq \tau<t_{i+1},
$$

for $0 \leq r \leq 2$, where $C_{3}$ depends on $M_{1}$.

Proof. It can be proved (see [10]) that for the Euler equation

$$
\begin{gathered}
\frac{\partial u}{\partial t}+(u \cdot \nabla) u+\nabla p=0, \\
\nabla \cdot u=0,
\end{gathered}
$$

there are constants $C_{4}, C_{5}$, such that if $t \leq \frac{1}{C_{4}\|u(0)\|_{3}}$, then $\|u(t)\|_{r} \leq C_{5}\|u(0)\|_{r}$ for $0 \leq r \leq 3$. By (58) and interpolation we have $\left\|\omega_{k}\left(t_{i}-0\right)\right\|_{2} \leq M_{1} k^{\frac{\sigma-3}{2}}$; hence $\left\|u_{k}\left(t_{i}-0\right)\right\|_{3} \leq C\left(M_{1}\right) k^{\frac{\sigma-3}{2}}$. Thus we take

$$
k_{0}=\left(\frac{1}{C_{4} C\left(M_{1}\right)}\right)^{\frac{2}{\sigma-1}}
$$

If $k \leq k_{0}$, then (60) holds.

Lemma 6.6. Under the assumptions of Lemma 6.5.

$$
\left(\sum_{i=0}^{i_{1}+1}\left\|\left(\omega^{*}-\omega_{k}\right)\left(t_{i}-0\right)\right\|_{\sigma}^{2} k\right)^{\frac{1}{2}} \leq C_{6} k^{\frac{5}{8}(\sigma-1)-2 \varepsilon},
$$

where $\varepsilon>0, k \leq k_{0}$, and $C_{6}$ depends on $M_{1}$.

Proof. Let $\tilde{F}=u \cdot \nabla \omega-\tilde{u}_{k} \cdot \nabla \tilde{\omega}_{k}$. We obtain by Lemma 6.1 and interpolation that

$$
\begin{aligned}
\|\tilde{F}\|_{-\frac{1}{2}+\varepsilon} & =\left\|\nabla \wedge\left(\nabla\left(\left(u-\tilde{u}_{k}\right) \otimes u+u \otimes\left(u-\tilde{u}_{k}\right)-\left(u-\tilde{u}_{k}\right) \otimes\left(u-\tilde{u}_{k}\right)\right)\right)\right\|_{-\frac{1}{2}+\varepsilon} \\
& \leq C\left\|u-\tilde{u}_{k}\right\|_{\frac{3}{2}+\varepsilon}+C\left\|u-\tilde{u}_{k}\right\|_{\frac{3}{2}+\varepsilon}^{2} \\
& \leq C\left\|\omega-\tilde{\omega}_{k}\right\|_{\frac{1}{2}+\varepsilon}+C\left\|\omega-\tilde{\omega}_{k}\right\|_{\frac{1}{2}+\varepsilon}^{2} \\
& \leq C\left\|\omega-\tilde{\omega}_{k}\right\|_{\frac{1}{2}+\varepsilon}+C\left\|\omega-\tilde{\omega}_{k}\right\|_{\sigma-1}\left\|\omega-\tilde{\omega}_{k}\right\|_{2-\sigma+2 \varepsilon} .
\end{aligned}
$$

Then we notice (58) and (60), to get for $\tau<t_{i_{1}+1}$ that

$$
\begin{aligned}
\|\tilde{F}\|_{-\frac{1}{2}+\varepsilon} & \leq C\left\|\omega-\tilde{\omega}_{k}\right\|_{2-\sigma+2 \varepsilon} \\
& \leq C\left\|\tilde{\omega}^{*}-\tilde{\omega}_{k}\right\|_{2-\sigma+2 \varepsilon}+C\left\|\omega-\tilde{\omega}^{*}\right\|_{2-\sigma+2 \varepsilon} .
\end{aligned}
$$

Since $\omega$ is sufficiently smooth, (49) and (51) imply that

$$
\left\|\omega(\tau)-\tilde{\omega}^{*}(\tau)\right\|_{\sigma-2} \leq C k^{\frac{7}{16}+\frac{3 \beta}{8}-\frac{\varepsilon}{2}}, \quad \tau<t_{i_{1}+1} .
$$

Because $\beta \in\left(0, \frac{1}{2}\right)$ and $\varepsilon>0$ are arbitrary, we take $\beta$ close to $\frac{1}{2}$ and $\varepsilon$ small; then the interpolation between it and (50) gives

$$
\left\|\omega(\tau)-\tilde{\omega}^{*}(\tau)\right\|_{2-\sigma+2 \varepsilon} \leq C k^{\frac{5}{8}(\sigma-1)-2 \varepsilon},
$$

and consequently

$$
\|\tilde{F}\|_{-\frac{1}{2}+\varepsilon} \leq C\left\|\tilde{\omega}^{*}-\tilde{\omega}_{k}\right\|_{2-\sigma+2 \varepsilon}+C k^{\frac{5}{8}(\sigma-1)-2 \varepsilon} .
$$


It follows from (36) that for $i \leq i_{1}+1$

$$
\begin{aligned}
\left(\omega^{*}-\omega_{k}\right)\left(x, t_{i}-0\right)= & -\sum_{j=0}^{i-1} \int_{\Omega} K\left(x-\xi, t_{i}-t_{j}\right) \int_{t_{j}}^{t_{j+1}} \tilde{F}(\xi, \tau) d \xi d \tau \\
& +\sum_{j=0}^{i-1} \int_{\partial \Omega} K\left(x-\xi, t_{i}-t_{j}\right) \int_{t_{j}}^{t_{j+1}}\left(\rho^{*}-\rho_{k}\right)(\xi, \tau) d s_{\xi} d \tau
\end{aligned}
$$

Then we get

$$
\begin{aligned}
\left\|\left(\omega^{*}-\omega_{k}\right)\left(t_{i}-0\right)\right\|_{\sigma} \leq & C \sum_{j=0}^{i-1} \int_{t_{j}}^{t_{j+1}}\left(t_{i}-t_{j}\right)^{-\frac{\sigma}{2}-\frac{1}{4}+\frac{\varepsilon}{2}}\|\tilde{F}\|_{-\frac{1}{2}+\varepsilon} d \tau \\
& +C \sum_{j=0}^{i-1} \int_{t_{j}}^{t_{j+1}}\left(t_{i}-t_{j}\right)^{-\frac{1+\sigma}{2}+\frac{\beta}{2}}\left\|\chi^{*}-\chi_{k}\right\|_{\beta} d \tau
\end{aligned}
$$

where $\sigma-1<\beta<\frac{1}{2}$. Analogously to (55), (56) and (59), we have

$$
\left\|\chi^{*}-\chi_{k}\right\|_{\beta, \frac{\beta}{2}, \Omega \times\left(0, t_{i}\right)} \leq C\|\tilde{F}\|_{L^{2}\left(0, t_{i} ; H^{-\frac{1}{2}+\varepsilon}(\Omega)\right)},
$$

where we notice that the term corresponding to $\omega_{0}$ is cancelled out.

Let $i_{2}$ be an index to be determined. We take the sum

$$
\begin{aligned}
& \sum_{i=0}^{i_{2}}\left\|\left(\omega^{*}-\omega_{k}\right)\left(t_{i}-0\right)\right\|_{\sigma}^{2} k \\
& \leq C \sum_{i=0}^{i_{2}}\left(\sum_{j=0}^{i-1} \int_{t_{j}}^{t_{j+1}}\left(t_{i}-t_{j}\right)^{-\frac{\sigma}{2}-\frac{1}{4}+\frac{\varepsilon}{2}}\|\tilde{F}\|_{-\frac{1}{2}+\varepsilon} d \tau\right)^{2} k \\
& +C \sum_{i=0}^{i_{2}}\left(\sum_{j=0}^{i-1} \int_{t_{j}}^{t_{j+1}}\left(t_{i}-t_{j}\right)^{-\frac{1+\sigma}{2}+\frac{\beta}{2}}\left\|\chi^{*}-\chi_{k}\right\|_{\beta} d \tau\right)^{2} k \\
& \leq C \sum_{i=0}^{i_{2}}\left(\sum_{j=0}^{i-1} \int_{t_{j}}^{t_{j+1}}\left(t_{i}-t_{j}\right)^{-\frac{\sigma}{2}-\frac{1}{4}+\frac{\varepsilon}{2}} d \tau\right) \\
& \cdot\left(\sum_{j=0}^{i-1} \int_{t_{j}}^{t_{j+1}}\left(t_{i}-t_{j}\right)^{-\frac{\sigma}{2}-\frac{1}{4}+\frac{\varepsilon}{2}}\|\tilde{F}\|_{-\frac{1}{2}+\varepsilon}^{2} d \tau\right) k \\
& +C \sum_{i=0}^{i_{2}}\left(\sum_{j=0}^{i-1} \int_{t_{j}}^{t_{j+1}}\left(t_{i}-t_{j}\right)^{-\frac{1+\sigma}{2}+\frac{\beta}{2}} d \tau\right) \\
& \cdot\left(\sum_{j=0}^{i-1} \int_{t_{j}}^{t_{j+1}}\left(t_{i}-t_{j}\right)^{-\frac{1+\sigma}{2}+\frac{\beta}{2}}\left\|\chi^{*}-\chi_{k}\right\|_{\beta}^{2} d \tau\right) k \\
& \leq C t_{i_{2}}^{\frac{3}{2}-\sigma+\varepsilon}\|\tilde{F}\|_{L^{2}\left(0, t_{i_{2}} ; H^{-\frac{1}{2}+\varepsilon}(\Omega)\right)}^{2}+C t_{i_{2}}^{1-\sigma+\beta}\left\|\chi^{*}-\chi_{k}\right\|_{L^{2}\left(0, t_{i_{2}} ; H^{\beta}(\Omega)\right)}^{2} .
\end{aligned}
$$


Then by (64)

$$
\sum_{i=0}^{i_{2}}\left\|\left(\omega^{*}-\omega_{k}\right)\left(t_{i}-0\right)\right\|_{\sigma}^{2} k \leq C t_{i_{2}}^{\alpha}\|\tilde{F}\|_{L^{2}\left(0, t_{i_{2}} ; H^{-\frac{1}{2}+\varepsilon}(\Omega)\right)}^{2},
$$

where $\alpha=\min \left(\frac{3}{2}-\sigma+\varepsilon, 1-\sigma+\beta\right)>0$.

Let us estimate the right hand side of (65) through (62). For $t_{i} \leq \tau<t_{i+1}$ we have

$$
\left\|\left(\tilde{\omega}^{*}-\tilde{\omega}_{k}\right)(\tau)\right\|_{2-\sigma+2 \varepsilon} \leq \int_{t_{i}}^{\tau}\|\tilde{F}\|_{2-\sigma+2 \varepsilon} d \tau+\left\|\left(\omega^{*}-\omega_{k}\right)\left(t_{i}-0\right)\right\|_{2-\sigma+2 \varepsilon}
$$

Then applying Lemma 6.1 we obtain

$$
\begin{aligned}
\|\left(\tilde{\omega}^{*}\right. & \left.-\tilde{\omega}_{k}\right)(\tau) \|_{2-\sigma+2 \varepsilon} \\
& \leq \int_{t_{i}}^{\tau}\left(C+C\left\|\tilde{u}_{k}\right\|_{1+\varepsilon}\left\|\tilde{\omega}_{k}\right\|_{3-\sigma+2 \varepsilon}\right) d \tau+\left\|\left(\omega^{*}-\omega_{k}\right)\left(t_{i}-0\right)\right\|_{2-\sigma+2 \varepsilon} .
\end{aligned}
$$

The inequalities (60) and (58) imply

$$
\left\|\left(\tilde{\omega}^{*}-\tilde{\omega}_{k}\right)(\tau)\right\|_{2-\sigma+2 \varepsilon} \leq C k^{\sigma-1-2 \varepsilon}+\left\|\left(\omega^{*}-\omega_{k}\right)\left(t_{i}-0\right)\right\|_{2-\sigma+2 \varepsilon} .
$$

We substitute (66) and (62) into (65), and obtain

$$
\begin{aligned}
& \sum_{i=0}^{i_{2}}\left\|\left(\omega^{*}-\omega_{k}\right)\left(t_{i}-0\right)\right\|_{\sigma}^{2} k \\
& \leq C t_{i_{2}}^{\alpha}\left\{\sum_{i=0}^{i_{2}-1}\left\|\left(\omega^{*}-\omega_{k}\right)\left(t_{i}-0\right)\right\|_{2-\sigma+2 \varepsilon}^{2} k+k^{\frac{5}{4}(\sigma-1)-4 \varepsilon}\right\} \\
& \leq C t_{i_{2}}^{\alpha}\left\{\sum_{i=0}^{i_{2}-1}\left\|\left(\omega^{*}-\omega_{k}\right)\left(t_{i}-0\right)\right\|_{\sigma}^{2} k+k^{\frac{5}{4}(\sigma-1)-4 \varepsilon}\right\} .
\end{aligned}
$$

We take $t_{i_{2}}$ small enough so that $C t_{i_{2}}^{\alpha} \leq \frac{1}{2}$, then get

$$
\sum_{i=0}^{i_{2}}\left\|\left(\omega^{*}-\omega_{k}\right)\left(t_{i}-0\right)\right\|_{\sigma}^{2} k \leq C k^{\frac{5}{4}(\sigma-1)-4 \varepsilon} .
$$

We notice that $t_{i_{2}}$ is independent of $k$; thus we can carry out an induction in a finite number, $T / t_{i_{2}}$, of steps. Suppose (61) is true for $i \leq N i_{2}$; then

$$
\begin{aligned}
& \sum_{i=N i_{2}+1}^{(N+1) i_{2}}\left\|\left(\omega^{*}-\omega_{k}\right)\left(t_{i}-0\right)\right\|_{\sigma}^{2} k \\
& \leq C \sum_{i=N i_{2}+1}^{(N+1) i_{2}}\left(\sum_{j=0}^{i-1} \int_{t_{j}}^{t_{j+1}}\left(t_{i}-t_{j}\right)^{-\frac{\sigma}{2}-\frac{1}{4}+\frac{\varepsilon}{2}}\|\tilde{F}\|_{-\frac{1}{2}+\varepsilon} d \tau\right)^{2} k \\
& \quad+C \sum_{i=N i_{2}+1}^{(N+1) i_{2}}\left(\sum_{j=0}^{i-1} \int_{t_{j}}^{t_{j+1}}\left(t_{i}-t_{j}\right)^{-\frac{1+\sigma}{2}+\frac{\beta}{2}}\left\|\chi^{*}-\chi_{k}\right\|_{\beta} d \tau\right)^{2} k
\end{aligned}
$$


By (62), (64) and the estimate for $i \leq N i_{2}$ we get

$$
\begin{aligned}
& \sum_{i=N i_{2}+1}^{(N+1) i_{2}}\left\|\left(\omega^{*}-\omega_{k}\right)\left(t_{i}-0\right)\right\|_{\sigma}^{2} k \\
& \leq C \sum_{i=N i_{2}+1}^{(N+1) i_{2}}\left(\sum_{j=N i_{2}+1}^{i-1} \int_{t_{j}}^{t_{j+1}}\left(t_{i}-t_{j}\right)^{-\frac{\sigma}{2}-\frac{1}{4}+\frac{\varepsilon}{2}}\|\tilde{F}\|_{-\frac{1}{2}+\varepsilon} d \tau\right)^{2} k \\
& \quad+C \sum_{i=N i_{2}+1}^{(N+1) i_{2}}\left(\sum_{j=N i_{2}+1}^{i-1} \int_{t_{j}}^{t_{j+1}}\left(t_{i}-t_{j}\right)^{-\frac{1+\sigma}{2}+\frac{\beta}{2}}\left\|\chi^{*}-\chi_{k}\right\|_{\beta} d \tau\right)^{2} k \\
& \quad+C k^{\frac{5}{4}(\sigma-1)-4 \varepsilon} .
\end{aligned}
$$

Following the same lines, we get the estimate for $i \leq(N+1) i_{2}$. After a finite number of steps we get the estimate up to $i \leq i_{1}+1$.

Lemma 6.7. Under the assumptions of Lemma 6.5,

$$
\left\|\left(\omega^{*}-\omega_{k}\right)\left(t_{i}-0\right)\right\|_{\sigma-1, \Omega} \leq C_{7} k^{\frac{5}{8}(\sigma-1)-2 \varepsilon},
$$

for $i \leq i_{1}+1$.

Proof. Analogously to (63), we have

$$
\begin{aligned}
\left\|\left(\omega^{*}-\omega_{k}\right)\left(t_{i}-0\right)\right\|_{\sigma-1} \leq & C \sum_{j=0}^{i-1} \int_{t_{j}}^{t_{j+1}}\left(t_{i}-t_{j}\right)^{\frac{1}{4}-\frac{\sigma}{2}+\frac{\varepsilon}{2}}\|\tilde{F}\|_{-\frac{1}{2}+\varepsilon} d \tau \\
& +C \sum_{j=0}^{i-1} \int_{t_{j}}^{t_{j+1}}\left(t_{i}-t_{j}\right)^{-\frac{\sigma}{2}+\frac{\beta}{2}}\left\|\chi^{*}-\chi_{k}\right\|_{\beta} d \tau,
\end{aligned}
$$

and then

$$
\begin{aligned}
\left\|\left(\omega^{*}-\omega_{k}\right)\left(t_{i}-0\right)\right\|_{\sigma-1} \\
\leq C\left(\sum_{j=0}^{i-1} \int_{t_{j}}^{t_{j+1}}\left(t_{i}-t_{j}\right)^{\frac{1}{2}-\sigma+\varepsilon} d \tau\right)^{\frac{1}{2}}\left(\sum_{j=0}^{i-1} \int_{t_{j}}^{t_{j+1}}\|\tilde{F}\|_{-\frac{1}{2}+\varepsilon}^{2} d \tau\right)^{\frac{1}{2}} \\
\quad+C\left(\sum_{j=0}^{i-1} \int_{t_{j}}^{t_{j+1}}\left(t_{i}-t_{j}\right)^{-\sigma+\beta} d \tau\right)^{\frac{1}{2}}\left(\sum_{j=0}^{i-1} \int_{t_{j}}^{t_{j+1}}\left\|\chi^{*}-\chi_{k}\right\|_{\beta}^{2} d \tau\right)^{\frac{1}{2}} .
\end{aligned}
$$

Then we use (62), (64) to get the desired estimate.

Theorem 6.1. If $1<\sigma<\frac{3}{2}$, then

$$
\left(\sum_{l=0}^{i}\left\|\left(\omega^{*}-\omega_{k}\right)\left(t_{l}-0\right)\right\|_{\sigma}^{2} k\right)^{\frac{1}{2}}+\left\|\left(\omega^{*}-\omega_{k}\right)\left(t_{i}-0\right)\right\|_{\sigma-1} \leq C k^{\frac{1}{4}}
$$

for $0 \leq i \leq\left[\frac{T}{k}\right]$, provided $k \leq k_{0}$, where $k_{0}>0$ is a fixed constant and $C$ is independent of $k$. 
Proof. We prove (58), (67) and

$$
\left(\sum_{l=0}^{i}\left\|\left(\omega^{*}-\omega_{k}\right)\left(t_{l}-0\right)\right\|_{\sigma}^{2} k\right)^{\frac{1}{2}} \leq C_{8} k^{\frac{5}{8}(\sigma-1)-2 \varepsilon}
$$

by induction. They are obviously satisfied for $i=0$.

Let us fix the constant $M_{0}$ in (57) first. By (50) it is easy to see that

$$
\left\|\tilde{\omega}^{*}\right\|_{L^{2}\left(0, T ; H^{\sigma-\varepsilon}(\Omega)\right)}+\left\|\tilde{\omega}^{*}\right\|_{\sigma-1-\varepsilon} \leq C_{9} .
$$

We take $M_{0}=2 C_{9}$.

We assume that (58), (67), and (69) are satisfied for $i \leq i_{1}$. Analogously to (666), we have

$$
\left\|\left(\tilde{\omega}^{*}-\tilde{\omega}_{k}\right)(\tau)\right\|_{\sigma-1} \leq C k^{\frac{1}{2}}+\left\|\left(\omega^{*}-\omega_{k}\right)\left(t_{i}-0\right)\right\|_{\sigma-1}
$$

for $\tau \in\left[t_{i}, t_{i+1}\right), i \leq i_{1}$. We substitute (67) in it and obtain

$$
\left\|\left(\tilde{\omega}^{*}-\tilde{\omega}_{k}\right)(\tau)\right\|_{\sigma-1} \leq C k^{\frac{5}{8}(\sigma-1)-2 \varepsilon} .
$$

(50), (60) and (58) imply

$$
\left\|\left(\tilde{\omega}^{*}-\tilde{\omega}_{k}\right)(\tau)\right\|_{\sigma} \leq C .
$$

Then by interpolation we obtain

$$
\left\|\left(\tilde{\omega}^{*}-\tilde{\omega}_{k}\right)(\tau)\right\|_{\sigma-\varepsilon} \leq C k^{\varepsilon\left(\frac{5}{8}(\sigma-1)-2 \varepsilon\right)} .
$$

We take $k_{0}$ small enough so that (57) holds for $k \leq k_{0}$ and $\tau<t_{i_{1}+1}$. Then from Lemma 6.4, Lemma 6.6, and Lemma 6.7 we conclude that (58), (67), (69) are satisfied for $i \leq i_{1}+1$. Thus the induction is complete. $\frac{1}{4}$.

We take $\sigma$ close to $\frac{3}{2}$ and $\varepsilon$ small enough. Then the exponent is greater than

Finally let us prove our main result, Theorem 1.1

Proof of Theorem 1.1. We take $\beta$ close to $\frac{1}{2}$ and $\varepsilon$ small enough in (48). Then

$$
\left\|\omega\left(t_{i}\right)-\omega^{*}\left(t_{i}-0\right)\right\|_{\sigma-2, \Omega} \leq C k^{\frac{1}{2}} \text {. }
$$

Theorem 4.1 implies

$$
\left\|\omega^{*}\left(t_{i}-0\right)\right\|_{\sigma, \Omega} \leq C
$$

Then by interpolation we get

$$
\left\|\omega\left(t_{i}\right)-\omega^{*}\left(t_{i}-0\right)\right\|_{\sigma-1, \Omega} \leq C k^{\frac{1}{4}}
$$

which in conjunction with (68) yields (23).

\section{A REMARK on the VORTEX SHEETS}

We have shown in (36) that the density of vortex sheets is $\int_{t_{j}}^{t_{j+1}} \rho_{k}(\xi, \tau) d \tau$. Let us make a heuristic analysis here to compare our result with the others in the literature.

The vorticity in (36) is divided into three parts: by initial data, by convection, and the vortex sheet, $\omega=\omega_{1}+\omega_{2}+\omega_{3}$. Suppose the velocity generated by $\omega_{1}+\omega_{2}$ is $u=u_{n} n+u_{\nu} \nu$ on the boundary $\partial \Omega$. Then $u_{n}=0$, due to the boundary condition of the Euler equation. The tangential component of velocity generated by the vortex sheets should be $-u_{\nu}$, to keep the no-slip boundary condition. 
On the other hand, potential theory tells us that for $x_{0} \in \partial \Omega$,

$$
\begin{aligned}
& \lim _{\substack{x \rightarrow x_{0} \\
x \in \Omega}} \int_{0}^{t} \int_{\partial \Omega} \frac{\partial}{\partial n_{x}} K(x-\xi, t-\tau) \rho_{k}(\xi, \tau) d s_{\xi} d \tau \\
= & \frac{1}{2} \rho_{k}\left(x_{0}, t\right)+\int_{0}^{t} \int_{\partial \Omega} \frac{\partial}{\partial n_{x}} K\left(x_{0}-\xi, t-\tau\right) \rho_{k}(\xi, \tau) d s_{\xi} d \tau .
\end{aligned}
$$

The left hand side is

$$
\left.\frac{\partial \omega_{3}}{\partial n}\right|_{\partial \Omega}=\frac{\partial}{\partial n}\left(-\frac{\partial \bar{u}_{n}}{\partial \nu}+\frac{\partial \bar{u}_{\nu}}{\partial n}\right)
$$

where $\bar{u}=\left(\bar{u}_{n}, \bar{u}_{\nu}\right)$ is generated by $\omega_{3}$. Since $\frac{\partial \bar{u}_{n}}{\partial n}+\frac{\partial \bar{u}_{\nu}}{\partial \nu}=0$, we have

$$
\left.\frac{\partial \omega_{3}}{\partial n}\right|_{\partial \Omega}=\frac{\partial^{2} \bar{u}_{\nu}}{\partial \nu^{2}}+\frac{\partial^{2} \bar{u}_{\nu}}{\partial n^{2}}=\triangle \bar{u}_{\nu}
$$

$\omega_{3}$ satisfies the heat equation on $\mathrm{R}^{2} \cdot \bar{u}$ satisfies the Stokes equation, but since $\nabla \cdot \bar{u}=0$ at $t=0, \bar{u}$ also satisfies the heat equation and the pressure equals to zero. We get

$$
\left.\frac{\partial \omega_{3}}{\partial n}\right|_{\partial \Omega}=\left.\frac{\partial \bar{u}_{\nu}}{\partial t}\right|_{\partial \Omega}=-\left.\frac{\partial u_{\nu}}{\partial t}\right|_{\partial \Omega} .
$$

Let us omit the second term in (170), to get $\left.\frac{\partial \omega_{3}}{\partial n}\right|_{\partial \Omega}=\frac{1}{2} \rho_{k}$. Then

$$
\rho_{k}=-\left.2 \frac{\partial u_{\nu}}{\partial t}\right|_{\partial \Omega}
$$

When we set a vortex sheet at $t=t_{j+1}$, we assume that the no-slip condition is satisfied at $t=t_{j}$. Therefore

$$
\int_{t_{j}}^{t_{j+1}} \rho_{k} d \tau=-2 \int_{t_{j}}^{t_{j+1}} \frac{\partial u_{\nu}}{\partial t} d t=-2 u_{\nu}\left(\xi, t_{j+1}\right) .
$$

We notice that $u_{\nu}\left(\xi, t_{j+1}\right)$ is the tangential component of velocity after the convection step. This is exactly the strength of vortex sheets given in some works.

By the above analysis the jump of velocity across the boundary is

$$
\begin{aligned}
{\left[u_{\nu}\right] } & =\int_{t_{j}}^{t_{j+1}}\left\{\lim _{x \rightarrow x_{0}, x \in \Omega^{c}} \frac{\partial \omega_{3}}{\partial n}-\lim _{x \rightarrow x_{0}, x \in \Omega} \frac{\partial \omega_{3}}{\partial n}\right\} d \tau \\
& =-\int_{t_{j}}^{t_{j+1}} \rho_{k}(\xi, t) d t=-2 u_{\nu},
\end{aligned}
$$

where $\Omega^{c}$ is the exterior of $\Omega$. Therefore it is assumed that $\left.u_{\nu}^{+}\right|_{\partial \Omega}=-\left.u_{\nu}^{-}\right|_{\partial \Omega}$, where "+" and " $-"$ indicate the exterior and the interior.

If $\Omega$ is a half plane, the second term of (70) is exactly zero. This observation coincides with that in 3. However, in general, this term does not vanish.

\section{ACKNOWLEDGMenT}

The author thanks the referees for their valuable comments. 


\section{REFERENCES}

[1] A.K.Aziz (ed.), The Mathematical Foundations of the Finite Element Method with Applications to Partial Differential Equations, Academic Press, New York and London, 1972. MR 49:11824]

[2] J.T.Beale, and A.Majda, Rates of convergence for viscous splitting of the Navier-Stokes equations, Math. Comp., 37, 243-259, 1981. MR 82i:65056

[3] G.Benfatto, and M.Pulvirenti, Convergence of Chorin-Marsden product formula in the halfplane, Comm. Math. Phys., 106, 427-458, 1986. MR 88a:35186

[4] A.J.Chorin, Numerical study of slightly viscous flow, J. Fluid Mech., 57, 785-796, 1973. MR 52:16280

[5] A.J.Chorin, T.J.R.Hughes, M.F.McCracken, and J.E.Marsden, Product formulas and numerical algorithms, Comm. Pure Appl. Math., 31, 205-256, 1978. MR 58:8230

[6] A.Pazy, Semigroups of Linear Operators and Applications to Partial Differential Equations, Springer-Verlag, 1983. MR 85g:47061

[7] R.Temam, On the Euler equations of incompressible perfect fluids, J. Funct. Anal., 20, 32-43, 1975. MR 55:3573

[8] R.Temam, Navier-Stokes Equations, Theory and Numerical Analysis, 3rd ed., North Holland, 1984. MR 86m:76003

[9] L.-A.Ying, Convergence of Chorin-Marsden formula for the Navier-Stokes equations on convex domains, J. Comp. Math., 17, 73-88, 1999. MR 2000d:65162

[10] L.-A.Ying, and P.Zhang, Vortex Methods, Science Press, Beijing/New York, and Kluwer Academic Publishers, Dordrecht/Boston/London, 1997. MR 2000f:76093

School of Mathematical Sciences, Peking University 100871, People's Republic of CHINA

E-mail address: yingla@pku.edu.cn 